\title{
Auction and Game-Based Spectrum Sharing in Cognitive Radio Networks
}

\author{
Dr. Omar Raoof and Prof. Hamed Al-Raweshidy \\ Brunel University-West London, \\ UK
}

\section{Introduction}

One of the main reasons behind the concurrent increase in the demand for and congestion of Radio Frequency (RF) spectrum is the rapid development of radio networks of all kinds in our world, which has defiantly changed the public feeling about radio. Nowadays, almost everybody has a mobile phone and radio stations are literary everywhere. Someone can argue that our world is becoming a radio world where waves are weaving everywhere around the Earth. What's more, this congestion has created a battle between the public, private and military sectors over frequency ownership and has put a premium on the cost of spectrum. According to a recent research introduced by the FCC (Federal Communications Commission) and Ofcom, it was found that most of the frequency spectrum was inefficiently utilized [1-2]. The existing spectrum allocation process, denoted as Fixed Spectrum Access (FSA), headed for static long-term exclusive rights of spectrum usage [3] and shown to be inflexible [4]. Studies have shown, however, that spectral utilization is relatively low when examined not just by frequency domain, but also across the spatial and temporal domains [5]. Thus, an intelligent device aware of its surroundings and able to adapt to the existing RF environment in consideration of all three domains, may be able to utilize spectrum more efficiently by dynamically sharing spectral resources [6 and 7]. Since the $19^{\text {th }}$ century, when the laws of electromagnetic have been discovered and described by the set of Maxwell's equations and technical devices been invented to produce and use these electromagnetic waves predicted by theory, man has added his own man-made waves to the natural ones [7].

It is fair to say that, from the very beginning of wireless telephony, maritime radio systems has always used shared channels [7-8]. For example, 2,182 KHz is used as a calling frequency as well as emergency signalling frequency and other frequencies are used as working frequencies. If two ships want to communicate, one should identify a working frequency and make a call. By specifying a channel or channels, that ships keep watch on, both emergency and establishing connections between ships can be facilitative. In fact, channel sharing was necessary and effective because of the lack of sufficient channels offered to every single ship and due to the fact that, the typical ship will require far less than a full channel of capacity [7-8]. Around the mid of 1970's, the FCC permitted land mobile operation on some of the lower UHF channels in several large cities, in order to expand land mobile services. One group of channels was made available to Radio Common Carriers (RCCs) to provide mobile service on a common carrier basis. The FCC adopted rules 
permitting open entry for these channels and requiring carriers to monitor the channels and select unused channel to carry each conversation. In essence, exclusivity was provided on a first come, first-served basis one conversation at a time [7-9].

Another example of spectrum sharing is the second generation of cordless telephone (CT2), developed by the British industry and government in the mid of 1980's. CT2 was designed to be used in both in home and in public and uses a pool of 40 channels. To establish a call, any equipment will automatically identify a vacant channel or a channel with the minimum interference and begins operation on that channel [7-8]. No one can ignore one of the main advantages of the radio, it can be used anywhere, at any time, capable of building links at very short distances as well as on a cosmic scale. Radio is a unique tool to connect men and things without any material medium. It is a wonderful tool for social progress. Having said all these facts about spectrum sharing, spectrum management can now be seen as a major goal for telecommunications efficiency. It is necessary that this natural and public resource be utilized for the profit of as many users as possible, taking care of the largest variety of needs.

If we want to talk about Cognitive Radio (CR), then we must mention Software Defined Radio (SDR), which is a transmitter in which operating parameters including transmission frequency, modulation type and maximum radiated or conducted output power can be altered without making any hardware changes. The sophistication possible in an SDR has now reached the level where a radio can possibly perform beneficial tasks that help the user, the network and help to minimize spectral congestion [7]. In order to raise an SDR's capabilities to make it known as a CR, it must support three major applications [7]:

Spectrum management and optimization.

Interface with a wide range of wireless networks leading to management and optimization of network resources.

Interface with human providing electromagnetic resources to aid the human in his and/or her activates.

We must begin with a few of the major contributions that have led us to today's CR developments, to truly recognize how many technologies have come together to drive CR technologies. The development of Digital Signal Processing (DSP) technologies arose due to the efforts of the research leaders [10-14], who taught an entire industry how to convert analog signal processes to digital processes. In the meantime, the simulation industry used in the radio industry was not only practical, but also resulted in improved radio communication performance, reliability, flexibility and increased value to the user [15-18].

The concept of CR emerged as an extension of SDR technology. Although, definitions of the two technology's are different, most radio expert agree with the fact that a CR device must have the following characteristic in order to be distinguished from an SDR one:

1. The named device should be aware of its environment.

2. The device must be able to change its physical behaviour in order to adapt to the changes of its current environment.

3. The device must be able to learn from its previous experience.

4. Finally, the device should be able to deal with situations unknown at the time of the device design. In another word, the device should be able to deal with any unexpected situations.

That being said, up to the authors knowledge, the idea of CR was first discussed officially in 1999 by [19]. It was a novel approach in wireless communications that the author describes 
it as "The point in which wireless personal digital assistants (PDA's) and the related networks are sufficiently computationally intelligent about radio resources and related computer-to-computer communications to detect user communications needs as a function of use context, and to provide radio resources and wireless services most appropriate to those needs." [19]. What's more, the work introduced in [19] can be considered one of the novel ideas which discussed CR technology. The work was based on the situation in which wireless nodes and the related networks are sufficiently computationally intelligent about radio resources and related computer-to-computer communication to detect the user communication needs as a function of use context and to provide resources and wireless resources most required. In another word, a CR is a radio that has the ability to sense and adapt to its radio environments. This work defined two basic characteristics of any CR device, which are cognitive capability and re-configurability. In order for the device to detect the spectrum parameters, the device should be able to interact with its environment. The spectrum needs to be analysed for spectrum concentration, power level, extent and nature of temporal and spatial variations, modulation scheme and existence of any other network operating in the neighbourhood. The CR device should be capable to adopt itself to meet the spectrum needs in the most optional method. The recent developments in the concept of software radios DSP techniques and antenna technology helped in this flexibility in CR devices design.

Finally, the intelligent support of CR's to the user arises by sophisticated networking of many radios to achieve the end behaviour, which provides added capability and other benefits to the user.

\section{Game theory and spectrum sharing}

Players in cooperative games try to maximize the overall profit function of everyone in the game in a fair fashion. This type of games has the advantage of higher total profit and better fairness. On the other hand, in non-cooperative or competitive games players try to maximize their own individual payoff functions. If such a game has a designer with preferences on the outcomes, it may be possible for the designer to decide on strategy spaces and the corresponding outcomes (i.e. the mechanism) so that the players' strategic behavior will not lead to an outcome that is far from desirable [20 and 21]. Recent studies have shown that despite claims of spectral insufficiency, the actual licensed spectrum remains unoccupied for long periods of time [8]. Thus, cognitive radio systems have been proposed [22] in order to efficiently exploit these spectral holes.

Previous studies have tackled different aspects of spectrum sensing and spectrum access. In [23], the performance of spectrum sensing, in terms of throughput, is investigated when the secondary users (SUs) share their instantaneous knowledge of the channel. The work in [24] studies the performance of different detectors for spectrum sensing, while in [25] spatial diversity methods are proposed for improving the probability of detecting the Primary User (PU) by the SUs. Other aspects of spectrum sensing are discussed in [26-27]. Furthermore, spectrum access has also received increased attention, e.g. [28-34]. In [28], a dynamic programming approach is proposed to allow the SUs to maximize their channel access time while taking into account a penalty factor from any collision with the PU. The work in [30] and [35-44] establishes that, in practice, the sensing time of CR networks is large and affects the access performance of the SUs. In [29], the authors model the spectrum access problem as a non-cooperative game, and propose learning algorithms to find the correlated equilibria 
of the game. Non-cooperative solutions for dynamic spectrum access are also proposed in [30] while taking into account changes in the SUs' environment such as the arrival of new PUs, among others.

Auctions of divisible goods have also received much attention [32] and [45-50]. Where the authors address the problem of allocating a divisible resource to buyers who value the quantity they receive, but strategize to maximize their net payoff (i.e. value minus payment). An allocation mechanism is used to allocate the resource based on bids declared by the buyers. The bids are equal to the payments, and the buyers are assumed to be in Nash equilibrium. When multiple SUs compete for spectral opportunities, the issues of fairness and efficiency arise. On one hand, it is desirable for an SU to access a channel with high availability. On the other hand, the effective achievable rate of an SU decreases when contending with many SUs over the most available channel. Consequently, efficiency of spectrum utilization in the system reduces. Therefore, an SU should explore transmission opportunities in other channels if available and refrain from transmission in the same channel all the time. Intuitively, diversifying spectrum access in both frequency (exploring more channels) and time (refraining from continuous transmission attempts) would be beneficial to achieving fairness among multiple SUs, in that SUs experiencing poorer channel conditions are not starved in the long run.

The objective of the work in this chapter is to design a mechanism that enables fair and efficient sharing of spectral resources among SUs. Firstly, we model spectrum access in cognitive radio networks as a repeated cooperative game. The theory and realization of cooperative spectrum sharing is presented in detail, where we assume that there is one PU and several SUs. We also consider the case of dynamic games, where the number of SUs changes. The advantages of cooperative sharing are proved by simulation. Secondly, we discuss the case of large number of SUs competing to share the offered spectrum and how the cooperative game will reduce the sellers and bidders revenue. Finally, we introduce a competitive auction and game-based mechanism to improve the overall system efficiency in terms of a better fairness in accessing the spectrum.

Throughout this chapter, an adaptive competitive second-price pay-to-bid sealed auction game is adapted as solution to the fairness problem of spectrum sharing between one primary user and a large number of secondary users in cognitive radio environment. Three main spectrum sharing game models are compared, namely optimal, cooperative and competitive game models introduced as a solution to the named problem. In addition, this chapter prove that the cooperative game model is built based on achieving Nash equilibrium between players and provides better revenue to the sellers and bidders in the game. Furthermore, the cooperative game is the best model to choose when the number of secondary users changes dynamically, but only when the number of competitors is low. As in practical situations, the number of secondary users might increase dramatically and the cooperative game will lose its powerful advantage once that number increases. As a result, the proposed mechanism creates a competition between the bidders and offers better revenue to the players in terms of fairness. Combining both second-price pay-to-bid sealed auction and competitive game model will insure that the user with better channel quality, higher traffic priority and fair bid will get a better chance to share the offered spectrum. It is shown by numerical results that the proposed mechanism could reach the maximum total profit for SUs with better fairness. Another solution is introduced in this chapter, which is done by introducing a reputation-based game between SUs. The game aims to elect one of the SUs to be a secondary-PU and arrange the access to other SUs. It is shown by numerical 
results that the proposed game managed to give a better chance to SUs to use the spectrum more efficiently and improve the PU revenue.

\section{Assumptions and system model}

\subsection{PU's and SU's and allocation function}

In the following sections, we consider a spectrum overlay-based cognitive radio wireless system with one PU and N SU's (as shown in Figure 6-1). The PU is willing to share some portion $\left(b_{i}\right)$ of the free spectrum $(F)$ with SU $i$. The PU asks each SU a payment of $c$ per unit bandwidth for the spectrum share, where $c$ is a function of the total size of spectrum available for sharing by the SU's. The revenue of SU $i$ is denoted by $r_{i}$ per unit of achievable transmission rate. A simple example is shown in Figure 1.

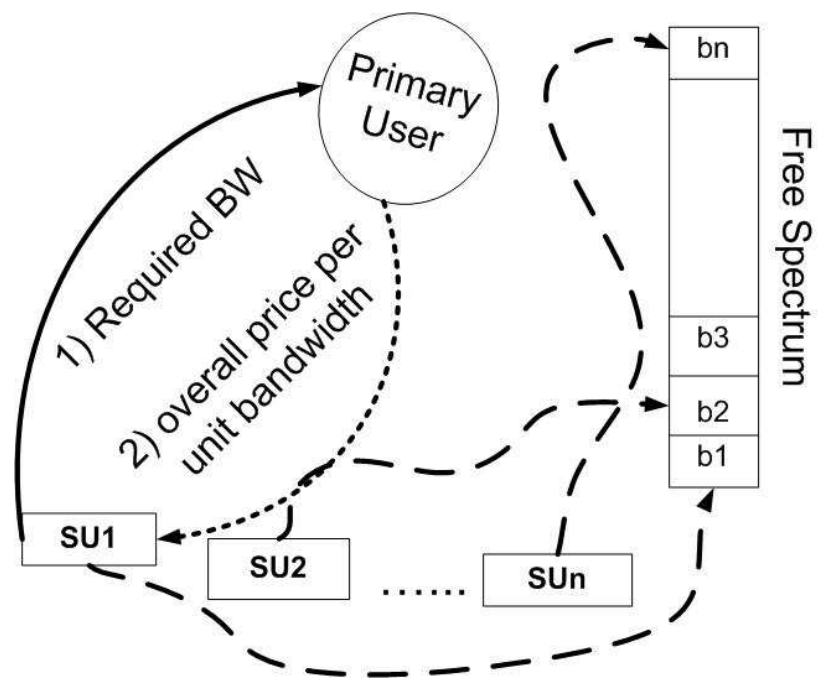

Fig. 1. System model for spectrum sharing.

Both centralized and distributed decision making scenarios are considers in this work. In the former case, each SU is assumed to be able to observe the strategies adopted by other users (i.e., either the users have the ability to discuss their shares between them, or the PU sends update of each SU share). In the latter case, the adaptation for spectrum sharing is performed in a distributed fashion based on communication between each of the SUs and the PU only (i.e., the secondary users are unable to observe the strategies and payoffs of each other).

\subsection{Cost function, and wireless system model}

A wireless transmission model based on adaptive modulation and coding (AMC) where the transmission rate can be dynamically adjusted based on channel quality is to be assumed in this chapter. With AMC, the signal-to-interference noise ratio (SINR) at the receiver is denoted as $\gamma$ and equals to;

$$
\gamma=\frac{p_{i} h_{i j}}{n_{0}+\Sigma_{j \neq i} p_{i} h_{i j}}
$$


Where $h_{i j}$ is the channel gain from the user $j$ 's transmitter to user $i$ 's receiver, $p_{i}$ is the transmitting power of the user $i$, and $n_{0}$ is the thermal noise level. The rate for user $i$ (in $\mathrm{bits} / \mathrm{sec} / \mathrm{Hz}$ ) is given by;

$$
R_{i}=\log _{2}(1+\gamma)
$$

The spectral efficiency $I_{S}$ of transmission by a secondary user can be obtained from [16];

$$
I_{s}=\log _{2}(1+K \gamma)
$$

Where $k=1.5 /\left(\ln 0.2 / B E R^{t a r}\right), B E R^{t a r}$ is the target bit-error-rate of the system. The pricing function [17] which the SU's pay is given by;

$$
c(F)=y\left(b_{1}+b_{2}+\ldots+b_{n}\right) z
$$

$y$ and $z$ are assumed to be positive constants and greater than one so that the function in convex (i.e., the function is continues and differentiable), knowing that $B$ is the set of bids for all SU's (i.e., $\left.\mathrm{B}=\left\{b_{1}, b_{2}, \ldots, b_{n}\right\}\right)$. Now let us denote $w$ as the worth of the spectrum to the PU. Then, the condition $c(F)>w \times \sum_{b j \in F} b_{j}$ must be satisfied in order to ensure that the PU is willing to share spectrum of size $b=\sum_{b_{j} \in F} b_{i} d_{j}$ with the SU's (if it is equal, then PU will not gain any profit).

The overall revenue of any SU can be explained as the combination of the user revenue of achievable transmission rate, the spectral efficiency and the shared portion of the spectrum (i.e., $r_{i} \times I_{S} \times b_{i}$ ). While the cost the user must pay is $b_{i} \times c(F)$. Then, the profit of every SU can be represented as;

$$
\mu_{i}=r_{i} \times I_{s} \times b_{i}-b_{i} \times c(F)
$$

The marginal profit of SU $i$ can be obtained from;

$$
\frac{d \mu_{i}(F)}{d b_{i}}=r_{i} I_{s}-y\left(\Sigma_{b_{j} \in F} b_{j}\right)^{z}-y z b_{i}\left(\Sigma_{b_{j} \in F} b_{j}\right)^{z-1}
$$

Knowing that, the optimal size of allocated spectrum to one SU depends on the strategies of other SU's are using. Nash equilibrium is considered as the solution of the game to ensure that all SU's are satisfied with it. By definition, Nash equilibrium of a game is a strategy profile with the property that no player can increase his payoff by choosing a different action, given the other players' actions. In this case, the Nash equilibrium is obtained by using the best response function, which is the best strategy of one player given others' strategies. Let $\mathrm{ST}_{-\mathrm{i}}$ denote the set of strategies adopted by all except $\mathrm{SU} i$ (i.e., $\mathrm{ST}_{-\mathrm{i}}=\left\{\mathrm{st}_{\mathrm{j}} \mid \mathrm{j}=1\right.$, $2, \ldots, N ; j \neq i\}$ and $\left.S T=S T_{-i} \cup\left\{s t_{i}\right\}\right)$. The best response function of SU $i$ given the size of the shared spectrum by other SU's $b_{j}$, where $j \neq i$, is defined as follows;

$$
B R_{i}=\arg \max _{b i} \mu_{i}\left(\mathrm{ST}_{-i} \cup\left\{b_{i}\right\}\right)
$$

Then the game is in Nash Equilibrium if and only if;

$$
b_{i}=B R_{i}\left(\mathrm{ST}_{-i}\right), \forall_{i}
$$




\section{Spectrum sharing strategies}

Cognitive radio is an intelligent wireless communication system that is aware of its surrounding environment and can be used to improve the efficiency of frequency spectrum by exploiting the existence of spectrum holes [22]. Spectrum management in cognitive radio aims at meeting the requirements from both the primary user and the secondary users. There are three strategies in spectrum sharing optimal, competitive and cooperative models.

\subsection{Optimal spectrum sharing model}

The objective of optimal model is to maximize the profit sum, which may make some secondary users have no spectrum to share [28, 32 and 51]. Therefore, it is unfair for all secondary users. From equation 6-6, the total marginal profit function for all the SU's can be denoted as follows: $\frac{d \sum_{j=1}^{N} \mu_{i}(F(t))}{d b_{i}(t)}$.

In order to get the solution of the biggest profit for all the secondary users, an optimal equation is built, as (6-9);

$$
\text { Maximize: } \sum_{j=1}^{N} \mu_{i}(F)
$$

Subject to: $\mathrm{b}_{\mathrm{i}} \geq 0, \forall b_{i} \in F$

Our assumption works as follow, the initial sharing spectrum is $b_{i}(0)$ for the $\mathrm{SU} i$, which is sent to the primary user. The PU adjusts the pricing function $c$, and then it is sent back to the SU. Since all secondary users are rational to maximize their profits, they can adjust the size of the requested spectrum $b_{i}$ based on the marginal profit function. In this case, each secondary user can communicate with the primary user to obtain the differentiated pricing function for different strategies. The adjustment of the requested/allocated spectrum size can be modelled as a dynamic game [49] as follows:

$$
b_{i}(t+1)=f\left(b_{i}(t)\right)=b_{i}(t)+\eta_{i} b_{i}(t) \frac{d \mu_{i}(F)}{d b_{i}(t)}
$$

Where $b_{i}(t)$ is the allocated spectrum size at time $t$ to SU $i$ and $\eta_{i}$ is the adjustment speed parameter (i.e., which can be expressed as the learning rate) of SU i. $f($.) denotes the selfmapping function. The SU can estimates the marginal profit function in the actual system by asking the price for share a spectrum from the PU of size $b_{i}(t) \pm \pi$, where $\Pi$ is a small number (i.e., $\Pi$ is 0.0001$)$. Simply after that the SU observes the response price from the PU $c^{-}($.$) and$ $c^{+}($.$) for b_{i}(t)-\pi$ and $b_{i}(t)+\pi$, respectively. Then, the marginal profits for the two cases $\mu_{i}-(t)$ and $\mu_{i}+(t)$ are compared and the marginal profit can be estimated from;

$$
\frac{d \mu_{i}(.)}{d b_{i}}=\frac{\mu_{i}^{+}(.)-\mu_{i}^{-}(.)}{2 \pi}
$$

The overall optimal profit can be estimated using equation (9).

\subsection{Competitive spectrum sharing model}

The main objective of competitive model is to maximize the profits of individual SU's by a game. The result is Nash equilibrium. In the distributed dynamic game, SU's may only be able 
to observe the pricing information from the PU; they cannot observe the strategies and profits of other SU's. The Nash equilibrium for each SU is built based on the interaction with the PU, similar to the case of the optimal sharing model. Since all SU's are rational to maximize their own profits, they can adjust the size of the requested spectrum $b_{i}$ based on the marginal profit function (i.e., equation (6)). In this case, each SU can communicate with the primary user to obtain different pricing function for different strategies. The adjustment of the requested/allocated spectrum size in competitive games show only a slight difference with optimal games, as each individual user is looking at improving his/her own profit. So equation (9) can be rewritten as;

\section{Maximize: $\mu_{i}(F)$}

Subject to: $b_{i} \geq 0, \forall b_{i} \in F$

In a similar way to the optimal game, an SU can estimate its marginal profit using the following equation:

$$
\frac{d \mu_{i}(F(t))}{d b_{i}(t)}=\frac{1}{2 \pi}\left\{\mu_{i}\left(F_{i}(t)+\pi\right)-\mu_{i}\left(F_{i}(t)-\pi\right)\right\}
$$

When $b_{i}(t+1)=b i(t)$ is satisfied, the Nash Equilibrium points $\left(b_{0}, b_{1}, b_{2}, \ldots, b_{N}\right)$ can be obtained.

\subsection{Cooperative spectrum sharing model}

As explained in previous section, in the model of competitive spectrum sharing, Nash equilibrium obtained at the maximum of the individual profit of SU. The result is not the best because they do not consider the interaction on other users. For cooperative spectrum sharing, the SU's can communicate with the consideration on the behaviour to other users. In this chapter, we assume that players can reach in common by communicating with each other. Decreasing the size of sharing spectrum a little for all the SU's on Nash equilibrium, (i.e., a factor $\sigma_{i}\left(0<\sigma_{i}<1\right)$ is multiplied on each SU strategy of Nash equilibrium). Although the size of shared spectrum has decreased, the cost which the PU charges to the SU decreases too, which results in the increase of the overall profit for all SU's and the total profits increase as well, but it might reduce the PU revenue.

SU's Nash Equilibrium strategy can be got from equation (10). All SU's will negotiate and multiply $\sigma_{i}$, the cooperative strategy is obtained $\left(i . e ., \sigma_{1} b_{1}, \sigma_{2} b_{2}, \ldots . ., \sigma_{N} b_{N}\right)$. $\sigma_{i}$ is chosen in such a way that both the overall and individual profit is maximized, which we called as the cooperative state;

$$
\text { Maximize: } \sum_{j=1}^{N} \mu_{i}(F) \text { and } \mu_{i}(F)
$$

Subject to: $b_{i} \geq 0, \forall b_{i} \in F$

However, we need to raise the problem of instability of this model. It is possible that one or more SUs may deviate from Nash equilibrium. For example, suppose $u 1$ to be the first SU to share the spectrum and want to deviate, its profit may increase by setting its marginal profit function of equation (6) to zero. If another SU $u 2$ does not change its strategy, the profit of $u 2$ will decrease. Therefore, any SU has the motive to deviate from cooperative state. In order to solve this problem, a mechanism needs to be applied to encourage the SUs not to deviate 
from the Nash state by computing the long term profit of the SU. Suppose SU $i$ is looking deviate from the Nash state, while SU $j(j \neq i)$ is still in the named state. Before SU $i$ deviate, it will compute the long term profit. The mechanism will multiply the future profit of SU $i$ (if decided to deviate) with a weight $\varepsilon_{i}\left(0<\varepsilon_{i}<1\right)$, which would make the profit in future stages are not higher than that of the previous stages, which means that the current profit is more valuable than future stages.

For any SU $i, \mu_{i}^{N s}, \mu_{i}^{N}, \mu_{i}^{d}$ denotes the profits of Nash state, Nash Equilibrium and deviation, respectively. There are two cases: one is that they all in Nash at all stages, no SU to deviate from the optimal solution, the long term profit of any SU $i$ is shown in equation (15). The other case is that $\mathrm{SU} i$ deviates from the optimal solution at the first stage, it will be in Nash equilibrium state in the following stages, and the long term profit of SU $i$ is shown in equation (16).

$$
\begin{gathered}
\mu_{i}^{N s}+\sigma_{i} \mu_{i}^{N s}+\sigma_{i}^{2} \mu_{i}^{N s}+\ldots=\frac{1}{1-\sigma_{i}} \mu_{i} \\
\mu_{i}^{d}+\sigma_{i} \mu_{i}^{N}+\sigma_{i}^{2} \mu_{i}^{N}+\ldots=\mu_{i}^{d}+\frac{\sigma_{i}}{1-\sigma_{i}} \mu_{i}
\end{gathered}
$$

The Nash state will be maintained if the long-term profit due to adopting the state is higher than that caused by deviation.

$$
\frac{1}{1-\sigma_{i}} \mu_{i}>\mu_{i}^{d}+\frac{\sigma_{i}}{1-\sigma_{i}} \mu_{i}
$$

i.e.,

$$
\sigma_{i} \geq \frac{\mu_{i}^{d}-\mu_{i}^{N s}}{\mu_{i}^{d}-\mu_{i}^{N}}
$$

From equation (15), we know that the Nash state will be kept because of low long term profit for the SU who wants to deviate. The weights $\sigma_{i}$ are the vindictive factors to inhabit the motive of leaving the cooperative state.

\section{Dynamic cooperative model}

In reality, the number of SUs may change. Sometimes there are more secondary users to apply for the spectrum offered by the primary user, and sometimes the secondary users have finished the communication and drop out of the spectrum as it has taken up. For example, let us suppose that there are two SUs, which have been in Nash state. Now there is another (new) SU to apply for the offered spectrum. We assume that the PU has no more spectrums to share. This will lead us to one solution, which is that the two SUs should make some of their spectrums exist to the newcomer.

During the process of reallocating, an adaptive method is applied with the following requirements. The total profit for all the SUs should be the biggest and it should be fair for the reallocation. Being prior users it is rational for them to have priority in spectrum allocation than those who comes later. In order to keep the total profit to maximum, those 
with better channel quality could take up more spectrum space. Therefore, the SUs with better channel quality could stop spectrum retreating earlier than those with worse channel quality. When the SUs reach optimal solution, the fairness will not be as good as the three SUs getting into Nash state directly. The reason is that these SUs coming at different time do not have the same priorities.

When SUs have finished the communication and exited the spectrum they had shared, an adaptive method is applied. A fixed part of the spectrum is allocated to the remaining SUs for each step. It is possible for SUs with better channel quality acquire more spectrum in order to make the total profit bigger.

\section{Simulation results}

\subsection{Static game (two SU's only in the game)}

In this section, we will consider a CR environment with one PU and two SUs sharing a frequency spectrum of $20 \mathrm{MHz}$ to $40 \mathrm{MHz}$. The system has the following settings; for the pricing function, $c(F)$, we use $y=1$ and $z=1$. The worth of spectrum for the PU is assumed to be one (i.e. $w=1$ ). The revenue of a SU per unit transmission rate is $r_{i}=10, \forall i$. The target average BER is $B E R^{t a r}=10^{-4}$. The initial value is $b_{i}(0)=2$. The adjustment speed parameter $\eta_{i}$ $=0.09$. The SNR for SUs $u_{1}$ and $u_{2}$ are denoted by $\gamma_{1}, \gamma_{2}$ where $\gamma_{1}=11 \mathrm{~dB}, \gamma_{2}=12 \mathrm{~dB}$.

\subsubsection{Optimal and competitive models}

As explained in the previous section, the total profit is represented by $\mu(B)=\mu_{1}(B)+\mu_{2}(B)$. In Figure 2 , the total profits in optimal model arrived at its biggest value 228.7333 when $\left(b_{1}\right.$, $\left.b_{2}\right)=(4.1,15.6)$.

The trajectories of optimal model and competitive model are shown in Figure 3, (with $\gamma_{1}$ $\left.=11 \mathrm{~dB}, \mathrm{Y}_{2}=12 \mathrm{~dB}\right)$, the initial value is $(2,2)$ for the two models. In competitive model, the

Total profit and spectrum share using Optimal Game

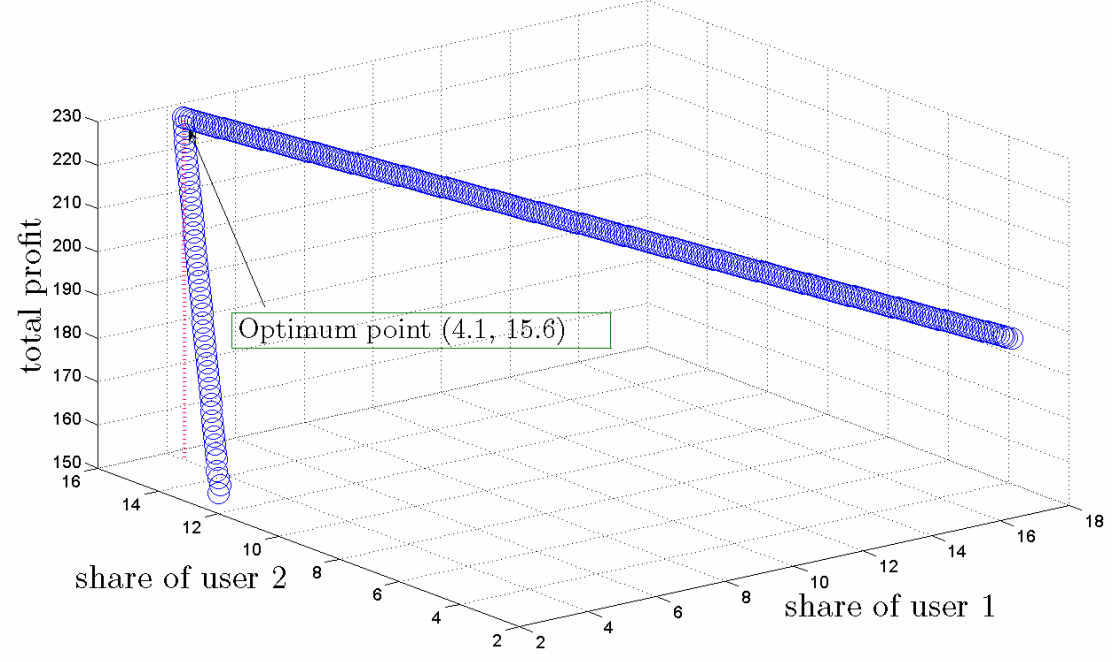

Fig. 2. Total profit and spectrum share using optimal game. 


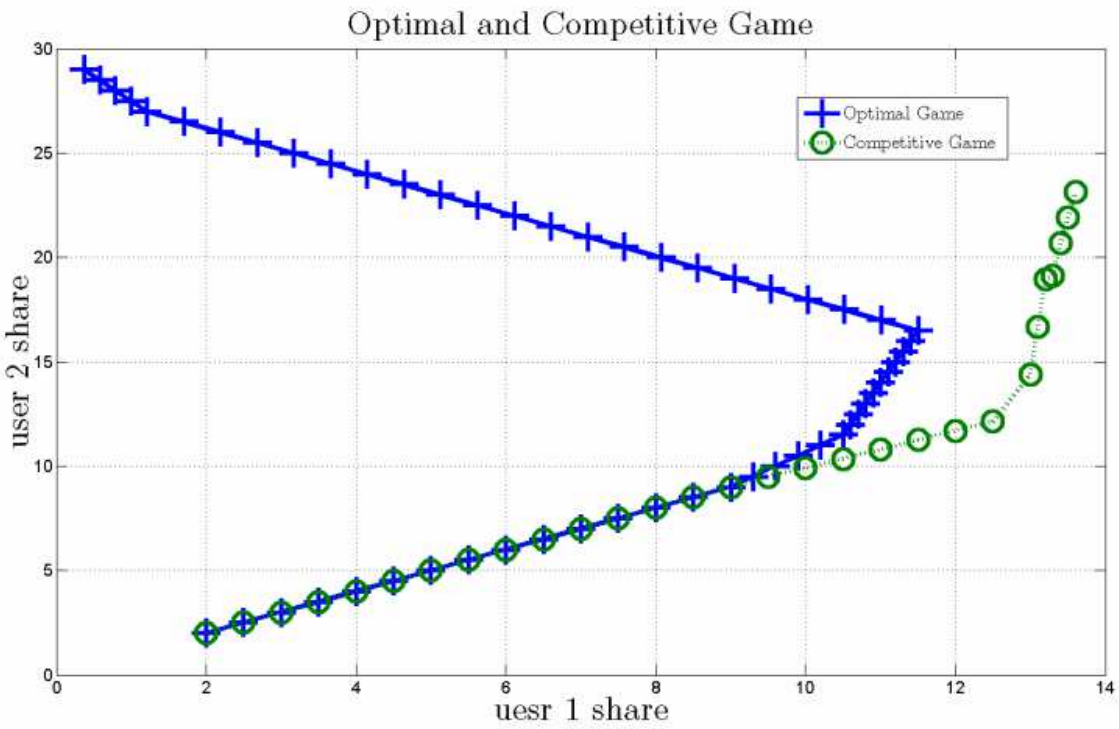

Fig. 3. Optimal and Competitive games

shared spectrum is determined by a game, where the two SUs have been in Nash equilibrium. In our simulation, the Nash equilibrium is at $(14.2591,24.1302)$. The sum of spectrum sharing is 11.3893 with the total profit of 228.2378 .

It can be seen that the total profit for optimal model is higher than that of competitive model obviously. But one SU has no spectrum sharing for the optimal model, which means the lack of fairness. The advantage of competitive model is fair with a lower profit sum.

\subsubsection{Cooperative spectrum sharing game}

Based on the Nash equilibrium, we set the weight $\sigma_{i}$ in the range of $[0.5,1]$. In order to keep the fairness, we assume $\left|\sigma_{1}-\sigma_{2}\right| \leq 1$ to guarantee the size of sharing spectrum is similar for both two SUs. Two SUs got their Nash equilibrium at $(18.2591,19.1302)$. At $\sigma_{1}=0.70, \sigma_{2}=0.80$, the total profit of 234.4963. Compared with the competitive model, we found that the shared spectrum in cooperative model is less than that of competitive model; it has a bigger total profit than that of Nash equilibrium, as shown in Figure 3.

The reason is that we set $\left(\sigma_{1} b_{1}, \sigma_{2} b_{2}\right)$ as the strategies to share the spectrum, the price is lower, and the total profit will increase. Now, let us suppose the SU $u_{1}$ deviates from the optimal solution. The strategy of SU $u_{2}$ does not change. SU $u_{1}$ adopts the strategy based on the marginal profit function. The profit for the two SUs will change when SU $u_{1}$ deviated. The comparison of the individual profit in cooperative model, competitive model and deviation is shown in Figure 4. The total profit for the SUs is shown in Figure 5. $\gamma_{1}$ is a variable, which changes in the range of $8 \sim 11 \mathrm{~dB}, \gamma_{2}=12 \mathrm{~dB}$.

It can be seen that $\mu_{1}, \mu_{2}$ are bigger in the cooperative model, compared with the competitive model. Therefore, the total profit is bigger too in the cooperative model. When SU $u 1$ deviates from the cooperative state, $\mu_{1}$ is higher, and $\mu_{2}$ is lower, and the total profit is lower (i.e. the amount of $\mu_{1}$ increasing is smaller than that of $\mu_{2}$ decreasing) as well. 


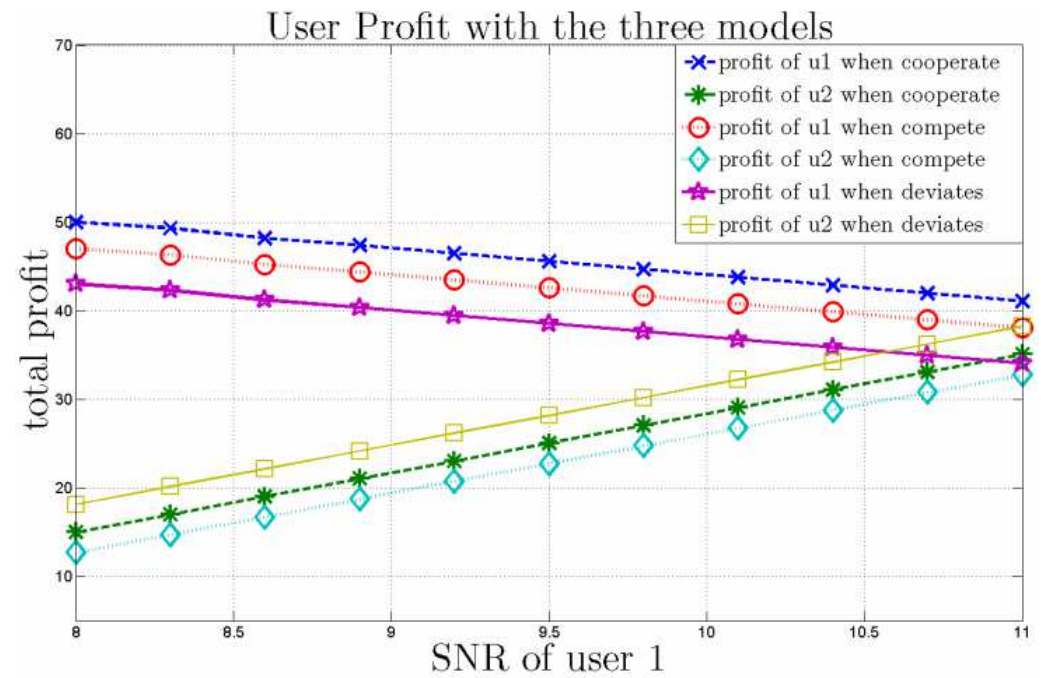

Fig. 4. Total profit with different modes.

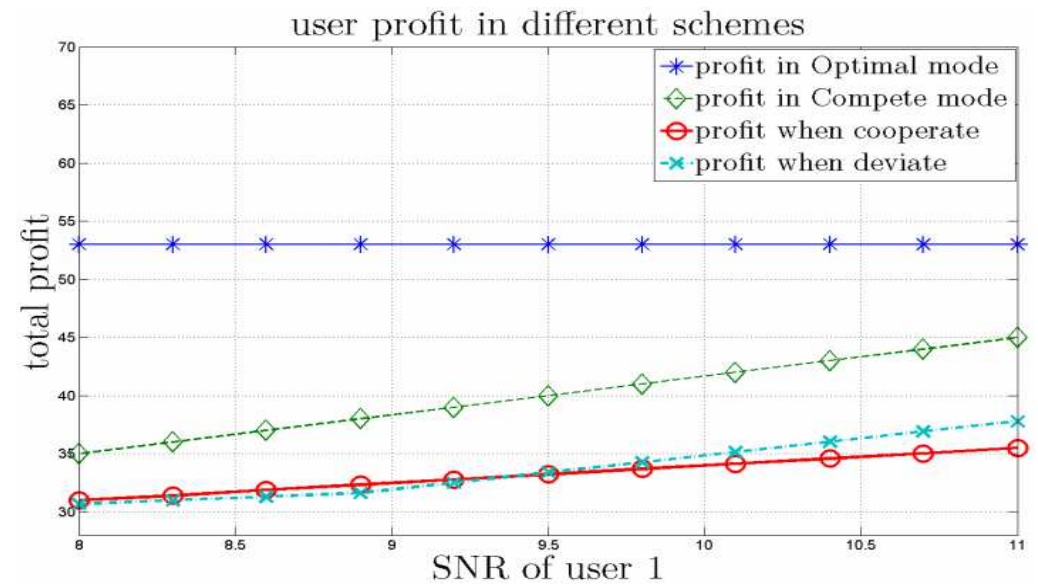

Fig. 5. User Profit with different modes.

\subsubsection{Dynamic spectrum sharing game}

The pervious results were based on the two SUs. The analyzing method is similar for more SUs. In practice, the number of SUs may change. For example, there is another secondary user denoted by $u_{3}$ looking to apply for the offered spectrum. We assume that the channel quality for $u_{3}$ is the same with secondary user $u_{2}\left(\gamma_{1}\right.$ is a variable, $\left.\gamma_{2}=\gamma_{3}=12 \mathrm{~dB}\right)$. There is no more free spectrum for the primary user to share with others. The previously mentioned adaptive method is applied in the allocation of spectrum. First $u_{1}$ and $u_{2}$ exit a fixed ratio of spectrum to $u_{3}$, and the total profit is computed. If the total profit could increase, the process will go on. If the total profit decreases, the SU with a better channel state will stop the process of exit. The trajectory of the process is shown in Figure 6. In addition, the 
corresponding total profit is shown in Figure 6-7. When a new SU applies for spectrum sharing, it would converge to the point of $(3.418948,5.4642,0.4936)$. The total profit is 62.3421, which is a little bigger than the case with two SUs. When the third SU exits the spectrum, an adaptive method is applied to reallocate the spectrum. The left two SUs converge to $(2.2148,5.9393)$ with a total profit of 73.9867 , as shown in Figure 6-8.

\section{Spectrum sharing in dynamic game}

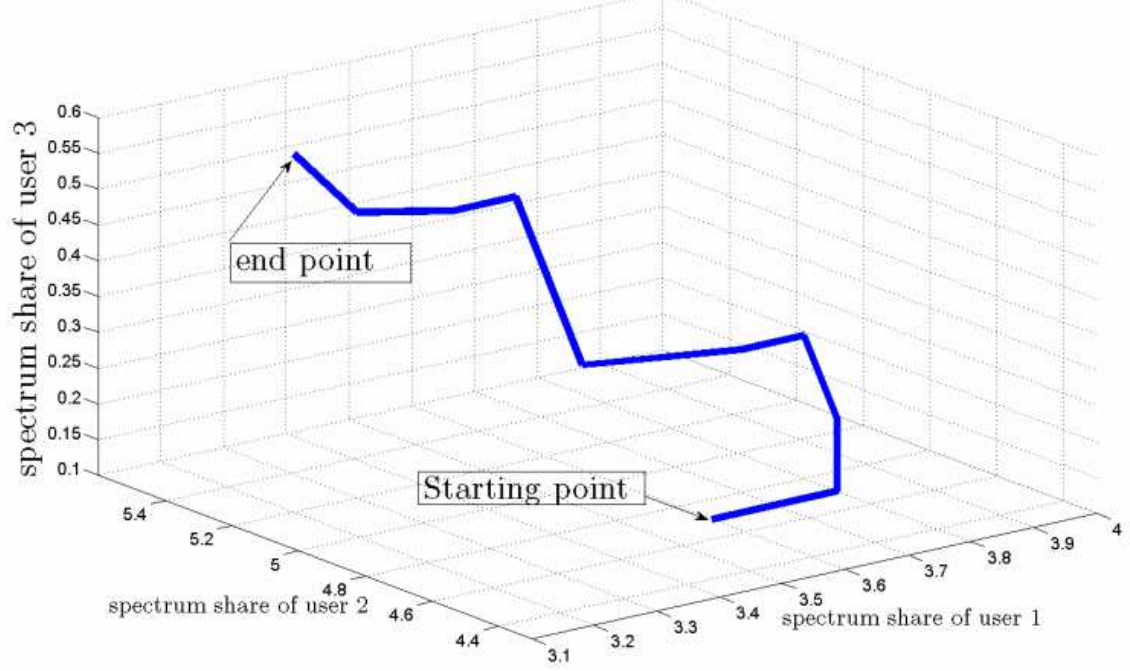

Fig. 6. Spectrum sharing in dynamic game.

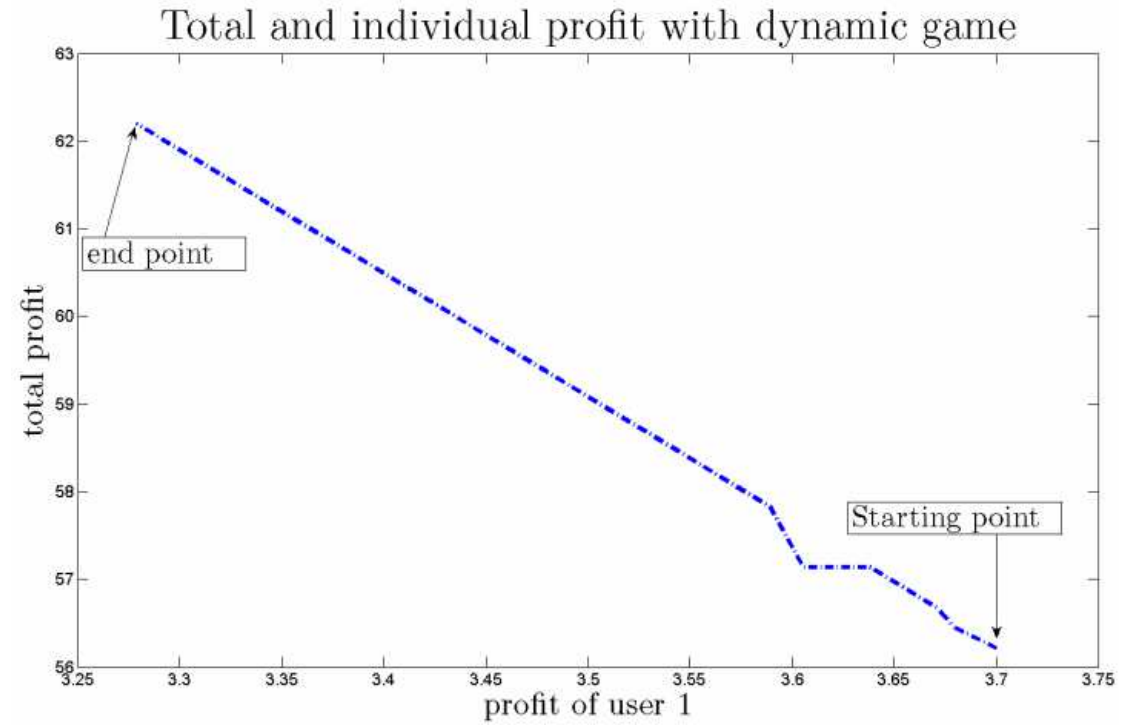

Fig. 7. Dynamic game and user profit. 


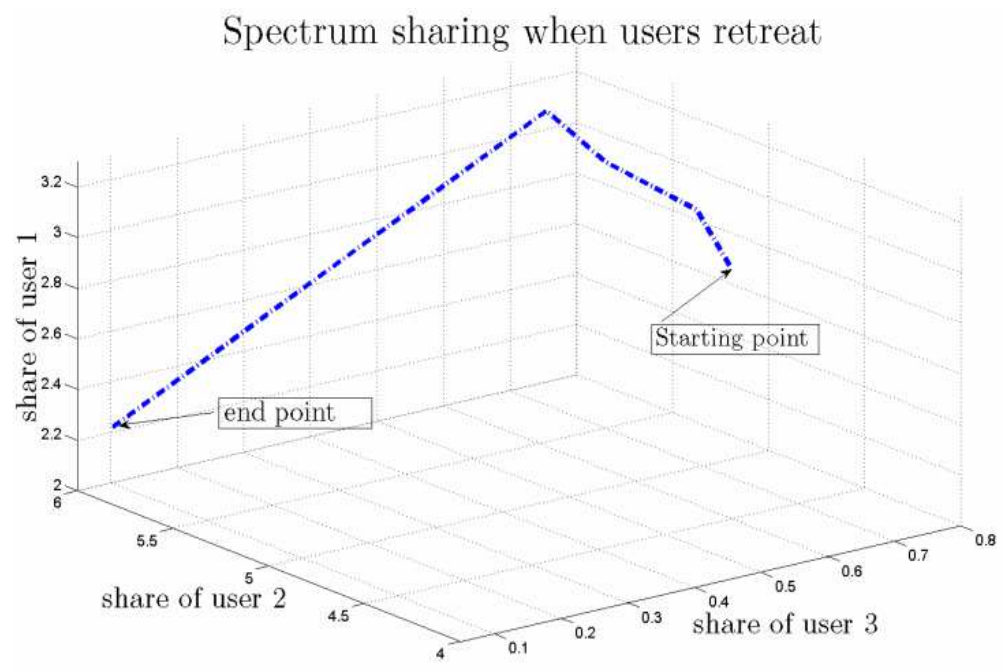

Fig. 8. Spectrum Share when user retreats.

\section{Is the cooperative game visible?}

So far we have discussed three game models to solve the problem of spectrum sharing in CR systems. We proved that the optimal game would improve the overall profit of the players in the game, which might lead to unfair distribution of the offered spectrum. The competitive game shows a lower overall profit, but gives a better share to the user with better channel quality, who ask for a share earlier and stays active for longer period (i.e., a higher priority as compared to new comers). Finally, the cooperative game gives the best overall individual profit and it is the best way to insure a fair share between multiple users in any CR system. However, does the cooperative game model works in an actual CR system?

In practical CR environment, the communication between competitors (i.e., players) is very hard to achieve. Individual users tend to contact the PU and ask for service [49], users can only observe the pricing function form the PU, but not the strategies and profits of other users. Nevertheless, achieving a cooperative scheme between the SUs (either, the PU forces the SU to get a fair share or using the model mentioned earlier) would improve both the seller and users revenue. Let us use the same assumption used in the previous section, where a PU have a $30 \mathrm{MHz}$ of free spectrum to offer to a group of users. The cooperative mode will work when the number of players is relatively small, so each player can discuss a fair share with the rest of the players. However, when the number of SUs increases, let say 20 or more SUs, the cooperative mode will not be useful anymore. If the PU or the users in such a scenario would decide to use the cooperative mode, the individual profit and share will be very low as compared to competitive game, taking into account the channel quality, user need and priority.

In order to solve such a problem, two solutions are proposed in the following sections. Firstly, a second-price pay-to-bid (or sometimes called as pay-as-bid) sealed auction mechanism is introduced to insure a fair competitive game between SUs. Secondly, 
reputation-based auction game is introduced as non-cooperative game to assign a SU to be a secondary-PU between other SUs. More details in the following sections:

\subsection{Pay-to-Bid competitive auction}

The allocation mechanism works as follows, let $W=\left[w_{1}, w_{2}, \ldots, w_{n}\right]$ be the non-negative bids (i.e., user valuation) that the SU will pay in order to get a share of the offered spectrum and let $X=\left[x_{1}, x_{2}, \ldots ., x_{n}\right]$ be the amount of the spectrum per unit bandwidth they are allocated as a result. We assume that the PU will announce the auction per unit bandwidth, for example the SUs will offer a bid for every $1 \mathrm{MHz}$ they will be allocated.

This allocation is made according to a cost-based allocation mechanism $\tau$, so that with the given payment $w$, the allocation to $\mathrm{SU} i$ is given by $x_{i}=\tau_{i}(w)$, as shown in Figure 6-9. $c$ will be assumed to be the reserved price of the PU, any SU bidding less than that will be withdrawn from the auction.

In order to reflect user $i$ 's valuation of the offered spectrum, a simple valuation function is proposed:

$$
v_{i}=I_{s} \times u p_{i}
$$

Where $v_{i}$ is user $i$ 's valuation to the offered spectrum per unit bandwidth, and $u p_{i}$ defines how much the user needs to get the desired share of the spectrum, which is a function of user traffic priority $\left(t p_{i}\right)$ and the channel SNR $\left(\gamma_{i}\right)$;

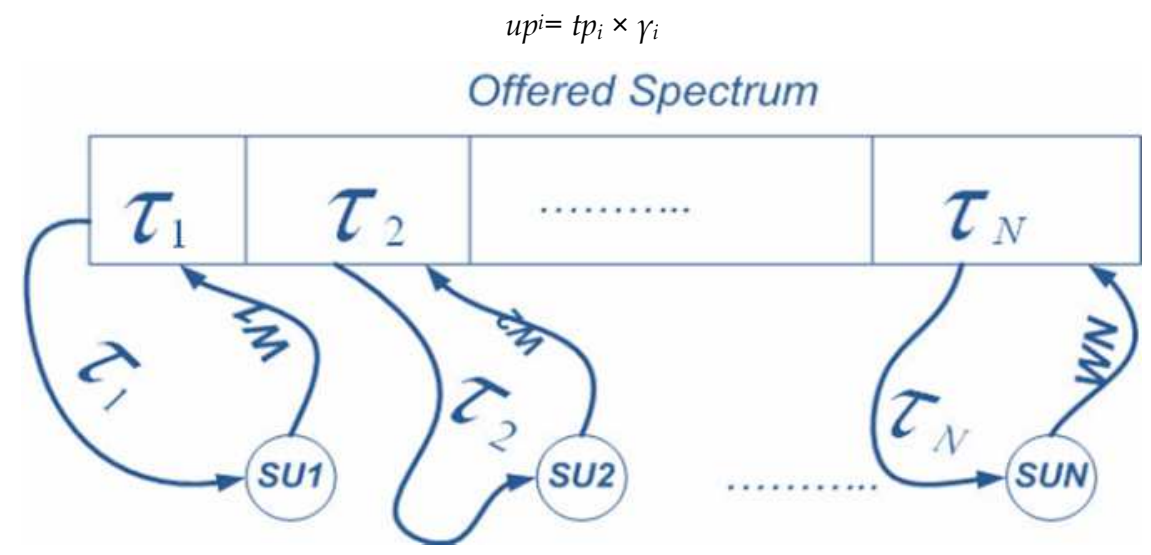

Fig. 9. Pay-to-bid allocation mechanism.

The user valuation can be interpreted that user $i$ uses the importance of his traffic and the channel quality (already known to all users) as a ruler to set his bid in the auction. This valuation measures the SU (if he wins the auction) capabilities to bid more for the offered spectrum keeping in mind the capacity of his channel. We can see that when the channel condition is good (according to equation (3)), the user will be more willing to increase his bid. As a result, a higher bid would be expected from him/her and vice versa.

We must mention that the auction mechanism is designed in such a way that $v_{i}$ does not represent the real price that an SU has to pay during the auction. Simply it is an interpretation of the strategic situation that a node is facing. In fact $v_{i}$ reflects the relationship between the user valuation and the channel condition. Additionally, since the 
channel coefficient $k$ is a random variable with a known distribution to each user, the distribution of the valuation $v_{i}$ is also known (according to their relationship shown in equation (16)). This means that $v_{i}$ lies in the interval $\left[v_{\min }, v_{\max }\right]$. We defined Bid as the bid space in the auction, $\left\{b i d_{1}, b i d_{2}, \ldots, b i d_{N}\right\}$, which represent the set of possible bids submitted to the PU. We can simply assign $b_{i d_{0}}$ to zero without loss of generality, as it represents the null bid. Accordingly, bid $d_{1}$ is the lowest acceptable bid, and $b_{i} d_{N}$ is the highest bid. The bid increment between two adjacent bids is taken to be the same in the typical case. In the event of ties (i.e. two bidders offer the same final price), the object would be allocated randomly to one of the tied bidders.

To find the winner of the first-price sealed-bid pay-to-bid auction, a theoretical model is defined based on the work of [52]. The probability of detecting a bid bid is denoted as $\xi_{1}$, the probability of not participating in the named auction will be denoted as $\xi_{0}$. Then the vector $\xi$, which equals to $\left(\xi_{1}, \xi_{2}, \ldots, \xi_{N}\right)$, denotes the probability distribution over Bid, where $\left(\sum_{i=0}^{N} \xi_{i}=\right.$ 1). Now we introduce the cumulative distribution function, which is used to find out whether a user $i$ will bid with $b_{i d}$ or less, $\sum_{j=0}^{i} \xi_{j}=\xi$, all of them are collected in the vector $\xi$. Then, any rational potential bidder with a known valuation of $v_{i}$ faces a decision problem of maximizing his expected profit from winning the auction; i.e.;

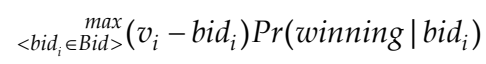

The equilibrium probability of winning for a particular bid $b_{i}$ is denoted as $\theta_{i}$, and these probabilities are collected in $\vartheta,\left(\vartheta_{0}, \vartheta_{1}, \vartheta_{2}, \ldots, \vartheta_{n}\right)$. Using $\xi$, the elements of the vector $\vartheta$ can be calculated. We can easily find that $\vartheta_{0}$ is known to be zero, as if any bidder submitted a null bid to the source, he is not going to win. We can calculate the remanning elements of $\vartheta$ as it can be directly verified that the following constitute a symmetric, Bayes-Nash equilibrium [53] of the auction game:

$$
\varphi_{i}=\frac{\xi_{i}^{n}-\xi_{i-1}^{n}}{n\left(\xi_{i}^{n}-\xi_{i-1}{ }^{n}\right)} \quad \forall i=0,1,2, \ldots, n
$$

We used the notation of Bayes-Nash equilibrium as defined in [53], there approach is to transform a game of incomplete information into one of imperfect information, and any buyer who has incomplete information about other buyers' values is treated as if he were uncertain about their types. From equation (21), we can see that the numerator is the probability that the highest bid is exactly equal to $b i d_{i}$, while the denominator is the expected number of users how are going to submit the same bid (i.e., bid $i_{i}$. For any user in the game, the best response will be to submit a bid which satisfies the following inequality;

$$
\left(v_{i}-b i d_{i}\right) \vartheta_{i} \geq\left(v_{j}-b i d_{j}\right) \vartheta_{j} \quad \forall j \neq i
$$

The above inequality shows that user $i$ 's profit is weakly beat any other user $j$ 's profit. The above inequality is the discrete analogue to the equilibrium first-order condition for expected-profit maximization in the continuous-variation model [52], which takes the form of the following ordinary differential equation in the strategy function $\varnothing\left(v_{i}\right)$;

$$
\mathscr{\emptyset}^{\prime}\left(v_{i}\right)+\varnothing\left(v_{i}\right) \frac{(n-1) f\left(v_{i}\right)}{F\left(v_{i}\right)}=v_{i} \frac{(n-1) f\left(v_{i}\right)}{F\left(v_{i}\right)}
$$


Where $f\left(v_{i}\right)$ and $F\left(v_{i}\right)$ are the probability density and cumulative distribution function of each bidder valuation respectively. We assume that they are common knowledge to bidders along with $n$, the number of bidders in the system. The reserve price is denoted by $c$, (In many instance, sellers reserve the right not to sell the object if the price determined in the auction is lower than some threshold amount [53], say $c>0$ ), and the above differential equation has the following solution;

$$
\varnothing\left(v_{i}\right)=v_{i}-\frac{\int v_{i} F(u)^{n-1} d u}{F\left(v_{i}\right)^{n-1}}
$$

In the case of the first-price sealed-bid auction, the bidder $i$ will submit a bid of $b i d_{i}=\varnothing\left(v_{i}\right)$ in equilibrium and he will pay a proportional price to his bid if he wins. On the other hand, for the second-price sealed-bid auction, a user $I$ will submit his valuation truthfully. This is because the price a user has to pay if he wins the auction is not the winning bid but the second highest one. Therefore, there is nothing to drive a user to bid higher or lower than his true valuation to the data offered by the server. In this case, $b i d_{i}=v_{i}$, shown in equation (18), and the payment process is the same as in the first-price auction. Once the winner has been announced, the PU will send an update message to all the SUs with the second highest price they need to pay in order to gain access. All SUs must pay the winning bid per unit bandwidth. To insure that the winner will get a higher priority than the rest of competitors, PU will send the winning bid to everyone and treat their replies according to the first bid was offered by the SUs in the first place.

This mechanism will offer a better competition in terms of fairness between players, the user with a better channel quality, a higher priority traffic and honest valuation will get a much better chance than other users to gain access to his/her desired share. Moreover, the named mechanism will improve the seller and winners revenue as compared to the optimal and cooperative game models.

Finally, next we will test the named mechanism with similar scenario assumptions as in the previous section. We are comparing three models; first, when the spectrum is offered to the users using a cooperative game. Second, using a similar setting but with a competitive game and finally a competitive second-price pay-to-bid sealed auction. We will study the effects in two simple scenarios; one, a SU (named $u_{1}$ ) who is competing with other bidders to get a share of the spectrum since the PU announce the auction. Two, a new comer is joining the game (the newcomer will join the game as the eleventh user onward) and how the introduced mechanism will improve his/her revenue, taking into account that the new comer has an excellent channel quality and a fair bid.

Figure 10, proofs what we discussed in section 6.1.3 in terms of individual user revenue. Although the cooperative games shows a better start (i.e., when the number of bidders is low), the cooperative game tries to improve the player's revenue and keep a fair share between all bidders. This would cause a sharp decrease in the seller revenue when the number of bidders increases. On the other the competitive game takes into account the channel condition and the user ability to grab his/her share before the others, that's why it shows better revenue when compared to the cooperative model.

For the second scenario, Figure 11 shows the dramatic improvement in the newcomer revenue; keeping in mind that his/her priority is rather high. Clearly, the introduced mechanism helped in improving spectrum share in terms of fairness, massively improving the players' revenue when compared to the other models and gives the PU a better deal by using the second-price sealed-auction. 


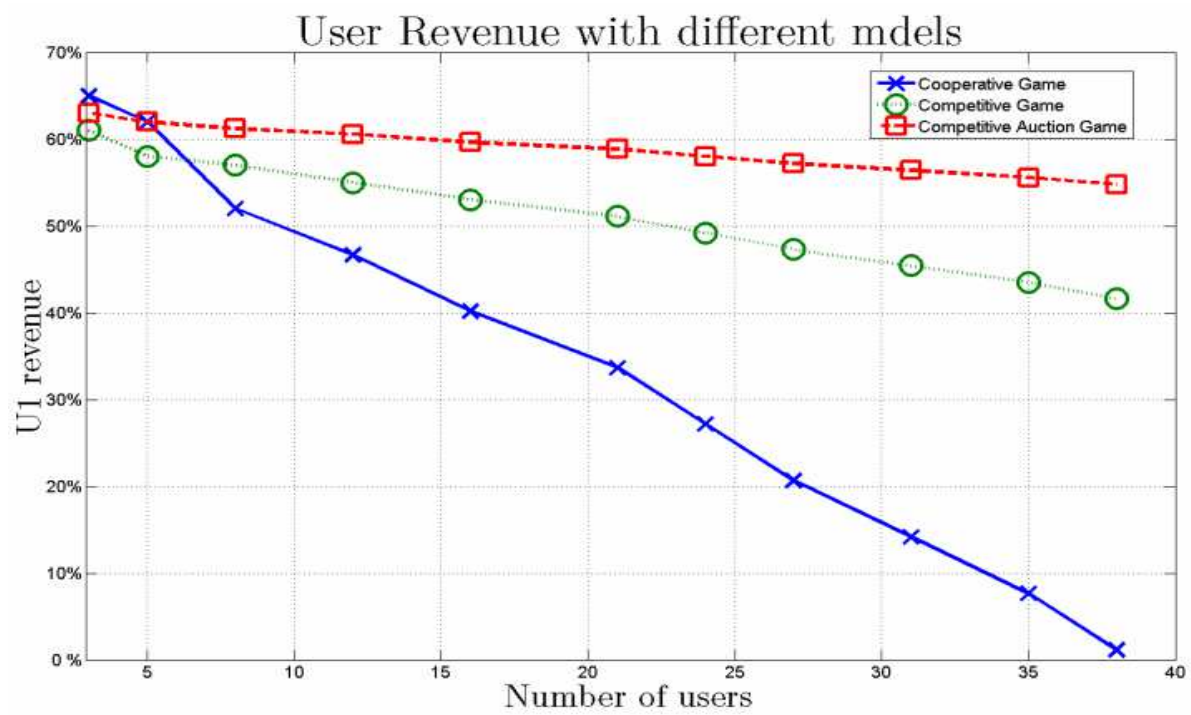

Fig. 10. SU revenue vs. number of users with different models.

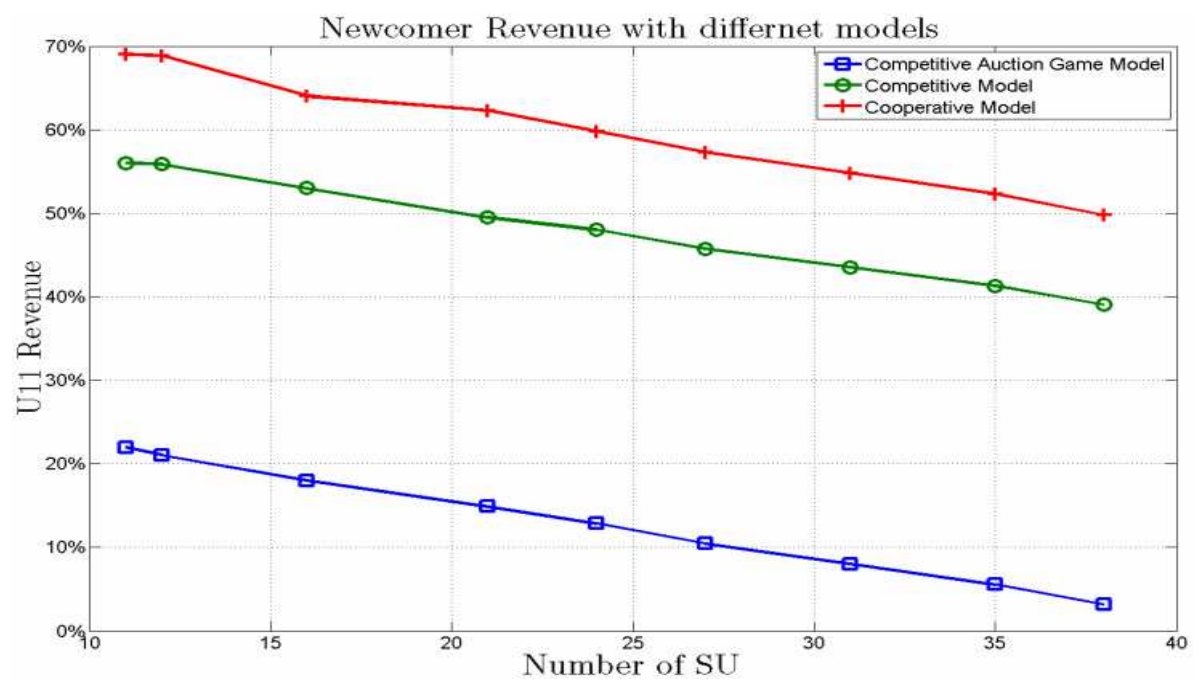

Fig. 11. Newcomer revenue vs. number of users.

\subsection{Reputation-based non-cooperative auction games}

With this game, PU will assign the spectrum to the winner of the second-price sealed auction process. The revenue of the PU will not change, as using the second-price auction insures that all bidders will bid around the real value of the offered spectrum. The winner of the auction will be a new PU between the rest of the SUs, and will have the right to decide whether to share the spectrum with the rest or not. However, a penalty factor is introduced 
to insure that not only paying more will guarantee a share of the spectrum but also reputation will be combined with each bid. This factor will be forwarded to the PU and will show whether the winner of the last auction was popular or not, which is done by helping other SUs to share the offered spectrum.

In this section we will represent the infinitely repeated version of game $G$ by $G^{\infty}$ (i.e. this is the case when $G$ is going to be played over and over again in successive time periods). We are assuming that the PU is offering a single frequency band to be shared by other SU's. However, if the PU is planning to offer more bands then the proposed mechanism must be repeated for the other bands between the secondary users. We will define the user reputation as $R$ which will depends on user performance during any time period $t$ as well as in prior time periods. Reputation of player $i$ in some time period $t$ is denoted by $R_{t}^{i}$. Formally, we define node reputation as follows:

$$
R_{t}^{i}=R_{t-1}^{i}(1-\propto)+w \times \propto \quad 0 \leq \propto \leq 1, \quad t \geq 2
$$

Where $\propto$ is the history of the user, it depends on the user reputation in the previous periods according to user behaviour. " $w$ " is equal to " 1 " when player $i$ at time $t$ is interested in sharing the offered spectrum and " 0 " otherwise. Therefore, $0 \leq R_{t}^{i} \leq 1$, i.e. the reputation value of each player varies between " 0 " and " 1 " (including) $\left(R_{t}^{i} \in[0,1]\right)$. Moreover, the reputation value of all players is equal to " 0 " when $t=0$. A high value of $\propto$ means the more importance is assigned to a player's need in sharing the spectrum with the PU (higher priority) during the current period than its previous need record, and vice versa. Thus, when $\propto$ is high, a user with even low reputation value in the current time period $t$, can significantly improve his/her reputation when it realises that it needs a better share of the spectrum.

As was defined the Nash equilibrium case earlier, the evaluation of the Nash equilibrium of the repeated game $G^{\infty}$ will be engaged. By finding the Nash equilibrium of $G^{\infty}$ it leads to the deduction of the Nash equilibria of G. The proposed incentive mechanism is based on a player's links reputation $R$. The benefit of which is that a player draws from the system to its contribution, the benefit is a monotonically increasing function of a player's contribution. Thus, this is a non-cooperative game among the players, where each player with high priority traffic wants to maximize his/her utility. The classical concept of Nash equilibrium points a way out of the endless cycle of speculation and counter-speculation as to what strategies the players should use. The intent is to deduce a symmetric Nash equilibrium because all the players belong to the same population/network (i.e., assume the same role) and it is therefore easier (i.e., require no coordination among players) to achieve such an equilibrium. If the players in a game either do not differ significantly or are not aware of any differences among themselves (i.e., if they are drawn from a single homogeneous population) then it is difficult for them to coordinate and a symmetric equilibrium, in which every player uses the same strategy, is more compelling.

The argument of a single homogeneous population implies that all the peers in a CR network have equivalent responsibilities and capabilities as everybody else. We assume that if the player chooses the action \{want to share\}, this will assign him a probability of $p$, and if the player chooses the action \{does not want to share\}, this will assign one a probability of $1-p$. 
It must be mentioned that in the action profile, a time and money saving Nash equilibrium case is defined, if all players choose the action \{does not want to share\}. As this will mean that, players are not interested in sharing the spectrum for the entire communication time. That is to say, users have low priority traffic and accessing the spectrum will be by chance, players will not compete to send their data and will not offer more money to the PU to get the spectrum. If any other player $i$ decided to switch to the action \{want to share\}, its payoff will be $-C$ which is less than a payoff of " 0 " that the node gets when decided not to share the spectrum. An undesirable Nash equilibrium case is generated, if all the players choose the action $\{$ want to share\}. This is easy to see because all nodes will have to compete against each other again, this will waste time and the winner will be the PU, as one of the SU's should pay more to share the offered spectrum.

The expected payoff of any player in period $t$ when it selects the action $\{$ want to share $\}$ is:

$$
p\left(-C+R_{t}^{\text {share }} \times U\right)
$$

This payoff is denoted as Payoff share, $U$ is the nodes utility. Similarly, the payoff for any player selects the action \{does not want to share\} will be:

$$
(1-p)\left(R_{t}^{\text {dontshare }} \times U\right)
$$

This will be denoted as payoff don'tshare. $_{\text {. It }}$ is easy to show that the term $R_{t}^{\text {share }} \times U$ captures the notation that the probability of SU becoming a secondary PU by sharing the offered spectrum is directly proportional to node's reputation.

$R_{t}^{\text {share }}$ is player $i$ reputation when he/she wants to share the offered spectrum at time $t$ (i.e. $w=1$ in equation (24)), and $R_{t}^{\text {don'tshare }}$ is player $i$ reputation when he/she decides to take the action $\{$ does not want to share $\}$ at the same time period $t$ (i.e. $w=0$ in equation (24)), from equation (24), we can get:

$$
R_{t}^{\text {share }}=R_{t-1}(1-\propto)+\propto
$$

and

$$
R_{t}^{\text {dontshare }}=R_{t-1}(1-\propto)
$$

Generally, each player's expected payoff in equilibrium is his/her expected payoff to any of its actions that he/she uses with positive probability. The above useful characterization of mixed-strategy Nash equilibrium yields to:

$$
\text { payoff } \text { share }=\text { payoff }_{\text {don'tshare }}
$$

Using equations 6-25, 6-26, and 6-27;

$$
p\left(-C+\left(R_{t-1}(1-\propto)+\propto\right) \times U\right)=(1-p)\left(R_{t-1}(1-\propto) \times U\right)
$$

Solving equation 9 to get the final value of $p$;

$$
p=\frac{R_{t-1} \times U \times(1-\propto)}{-C+2 R_{t-1} \times U \times(1-\propto)+U \times \propto}
$$


It must be mentioned that the value $p$ obtained above is not a constant, but varies in each time interval depending upon a node's reputation at the end of the previous time interval $t-1$.

Finally, the mixed strategy pair $(p, 1-p)$ for actions $\{$ want to share, does not want to share $\}$ respectively, is a mixed strategy Nash equilibrium for the players (i.e. nodes in the network). Assuming no collusion among nodes, if all the other nodes follow the above strategy, then the best strategy for any node is to also to follow one of the above strategies. Actually, this is a symmetric mixed strategy Nash equilibrium for any $G$, as well as $G^{\infty}$. In fact, it is a more stable equilibrium than the one in which no node is interested in sharing the offered spectrum. This is caused by two reasons. First, when none of the SUs is interested in sharing the spectrum, the network is not useful to any user. Second, in real-time scenarios, users that derive finite utility from altruism would always send some messages irrespective of how much they obtain in return. Therefore, it is unlikely to have a scenario in which no node is looking to contact the PU to share the spectrum.

\subsection{Properties of the proposed Nash Equilibrium}

In this section, we will present some of the interesting properties of the Nash equilibrium derived in the section above

\subsubsection{Simplicity of calculating the Nash Equilibrium}

In section '6.7.2', we have calculated the probability of achieving the equilibrium point between the SUs. This was based on which node will decide to share the spectrum with the PU and become a secondary PU. In each round of the game (or time period $t$ ) players decide whether they should ask to share the offered spectrum or not, based on their reputation at the end of the prior time period. This probability, as one can see, does not remain constant from one period to another. Moreover, it depends on a player's reputation at the end of the last time period. Players can calculate their reputation using equation (24), since they know precisely their actions at each round of the game. Thus, determining the Nash equilibrium strategy is fairly straightforward for any player. However, it must be noted that there is an inherent assumption that nodes are serviced based on their current reputation.

Figure 12, shows how players' reputations change in every time interval depending on their Nash strategy. At the beginning of the communication time, both, player 1 and 2 are competing with each other to guarantee access to the offered spectrum. However, player 1 uses the spectrum but at the same time managed to help player 2 (i.e. player 1 will be the secondary PU and will manage the access of players 2 and 3 to the offered spectrum). Player 3 shows his interest in the offered spectrum after the third time interval, and managed to use the spectrum once both player 1 and 2 finished using it or they are not interested anymore in sharing it. The figure shows the players (nodes) reputation values $0 \leq R_{t}^{i} \leq 1$ over ten time intervals.

On the other hand, Figure 13 below shows the same result but over a longer time period, around nine hundred time intervals. Similarly, three nodes are competing with each other, player one with the highest reputation and player three with the lowest. Player 1 will act as the secondary PU over the other two users (i.e. player 2 and 3). In this figure we used a random matrix generator to show different reputations when player 1 is interested to share the spectrum for $80 \%$ of the time, player 2 for $50 \%$ of the time and player 3 for $8 \%$ of the time only. 


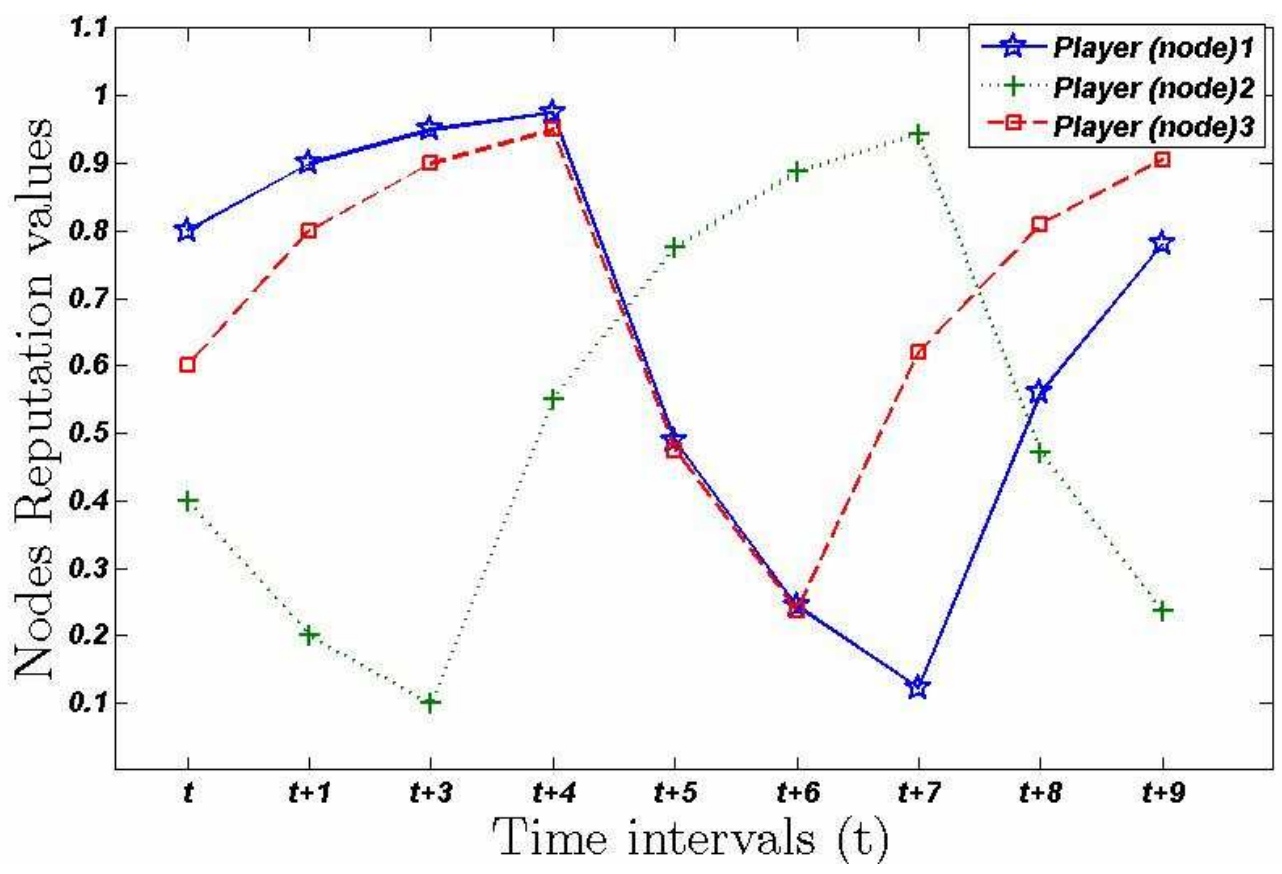

Fig. 12. Change in player's reputation controlled by their Nash equilibrium strategies.

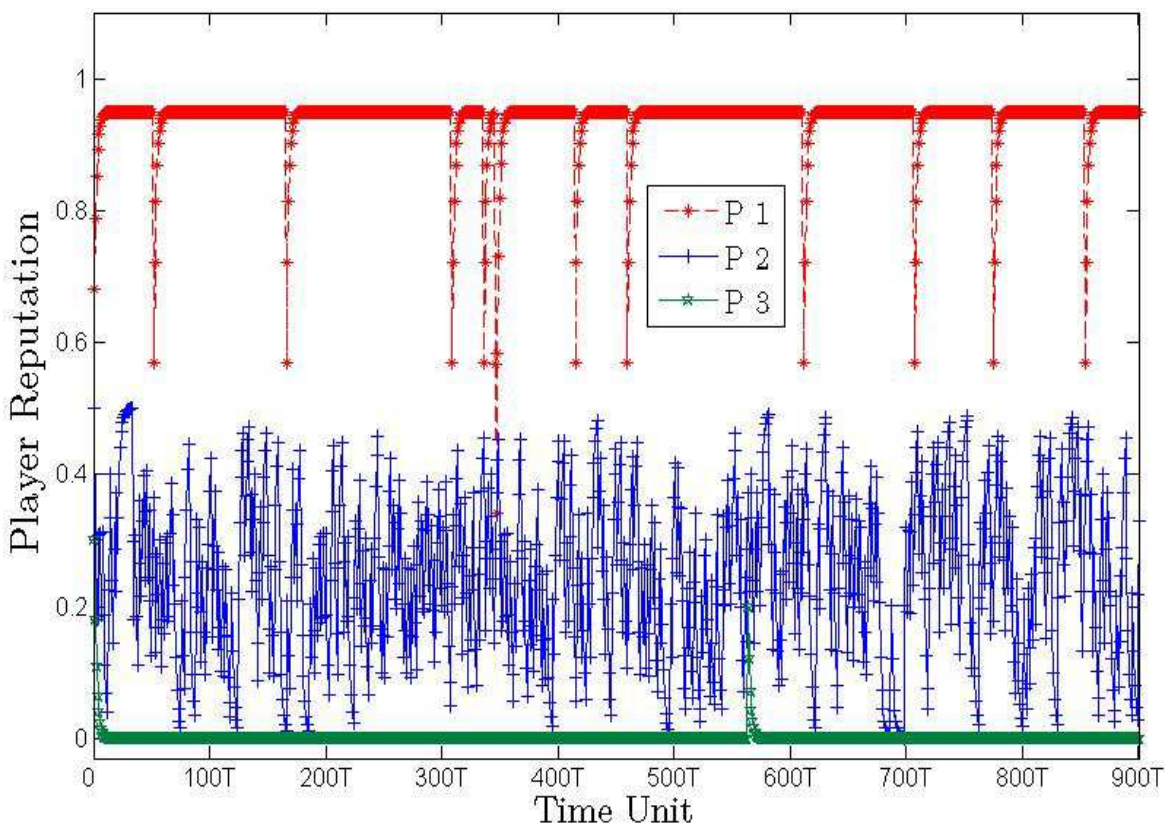

Fig. 13. Changing player reputation over a longer time period. 


\subsubsection{Addressing the spectrum to the right user}

The simple game theoretic model presented in the previous sections, wherein node reputation is used as a basis for deciding who will share the offered spectrum, predicts that it is in every peer's best interest to serve others. This includes the nodes that are not interested to share the spectrum at the current time period. Our simulations support this behaviour as we found that the total service received by a node is balanced by the total service that it has to offer to others, as shown in Figure 12.

\subsubsection{Addressing the problem of competitive sharing}

An important property of the equilibrium emerges from equation (30) that predicts the probability with which one node will be a secondary PU and it should serve others. If we set the value of $C$ in away such that, $C \ll<U$ (i.e. $C$ can be ignored from equation (6-30)), then equation (6-30) becomes:

$$
p=\frac{R_{t-1}(1-\propto)}{2 R_{t-1}(1-\propto)+\propto}
$$

That would lead us to the conclusion that $p<0.5$. Then, Nash equilibrium of the proposed game predicts that players should help each other less than 50 percent of the time when PU offers the spectrum. This, although it appears to be very restrictive, is a consequence of the fact that all nodes are selfish and are better off trying to share the spectrum than serving others. Intuitively, if a node knows that everyone else in the network behaves selfishly, i.e., provide as little service as possible, then the best strategy for the named node cannot be to serve others most of the time (i.e., with probability greater than 0.5 ).

\subsubsection{Fairness and equal sharing of cost and spectrum}

We concluded from the previous section that serving with a priority of less than 50 percent (i.e. when $C<<<U$ ) is an optimal point, the observer can notice that the overall system efficiency is severely reduced. This is because most of the nodes in the network act selfishly and at least half of the service requests from other nodes are not fulfilled. On the other hand, this equilibrium strategy provides fairness in the sense that the cost of system inefficiency is not burn by a single node (i.e. has one positive side), but it is shared among all nodes. This is because each node's request is likely to be turned down by the serving node (i.e. selfish secondary PU). In this work, we assume that if a node's request at one node is turned down, the node tries at some other candidate node capable of serving the request. On average, the probability that a node's request is successfully served in a time period is proportional to its current reputation.

\subsubsection{Decreasing $\alpha$ for a better share of the spectrum}

Figure 14 shows the effects of $\propto$ on the reputation probability of the nodes in the case where the node is not interested in sharing the spectrum. On the other hand, the node in figure 15 is looking to keep its share of the spectrum (derived from equation (27)).

As can be seen from Figures 14 and 15, a lower value of a shifts the reputation probability curve upwards. However, that all depends on whether the node is interested in using the offered spectrum or not. If the node is looking to give its share of the spectrum to other nodes, a low value of $\propto$ will gradually help the node to lose its share, however a high value of $\propto$ will guarantee a faster release of the spectrum. This is true for Figure 15 as well, which 


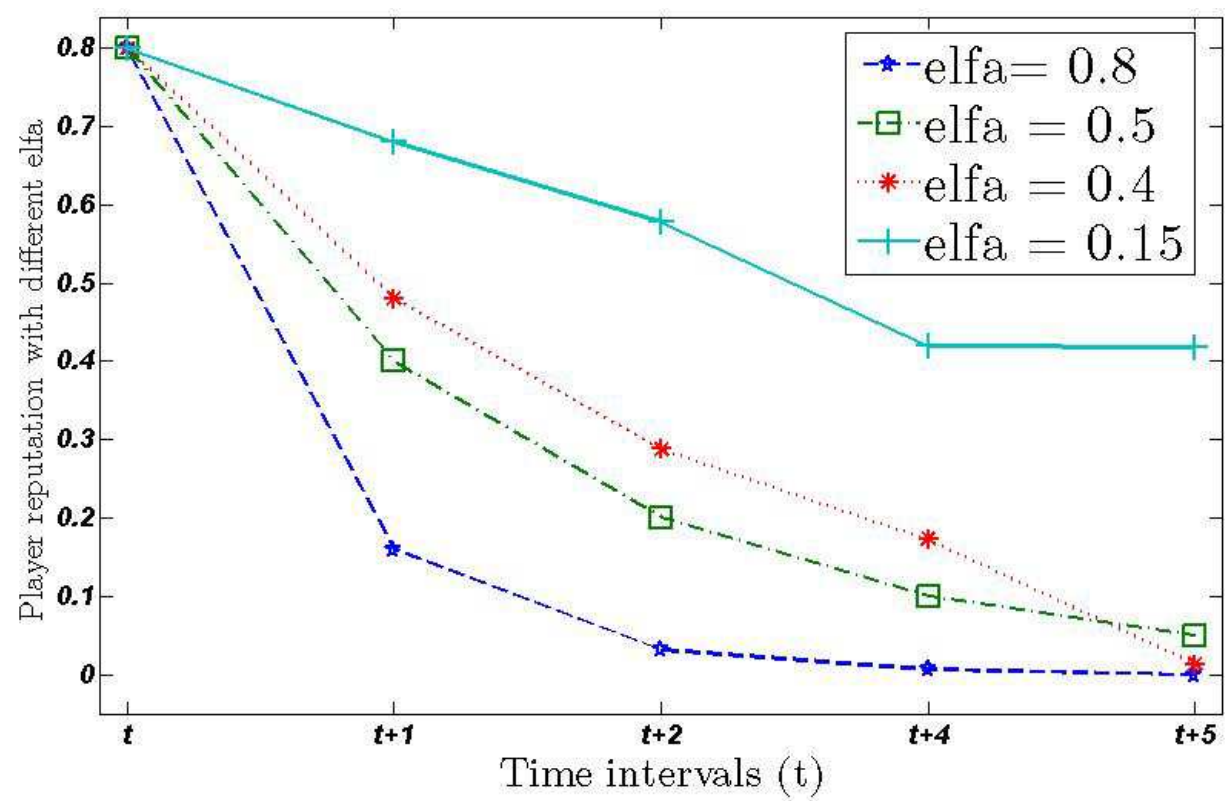

Fig. 14. Players reputation with respect to $a$ and the node is not interested in sharing the offered spectrum.

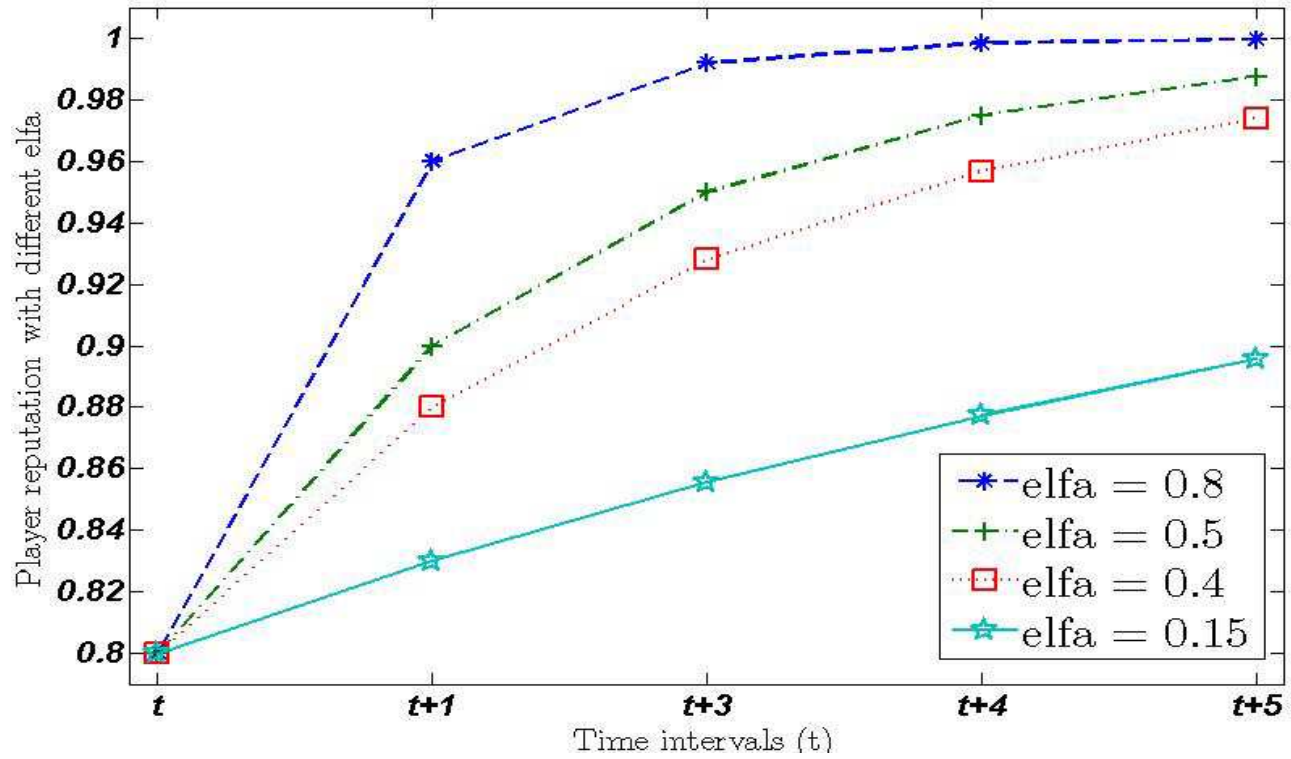

Fig. 15. Players reputation with respect to a and the node is definitely interested in sharing the offered spectrum from the PU. 
is to be expected since $\propto$ determines how much importance is given to a node's current performance as compared to its past service record. A low value of $\propto$ (i.e., giving more importance to nodes past actions up to the current time period $t$ ) means that nodes need to continually provide service to be able to maintain high reputation and access spectrum offered from the PU. If however $\propto$ is high, nodes can easily increase their reputation in any period in which they provide service to other nodes. This is irrespective of how cooperative they have been in the past with regards to providing service to others. Therefore a simple way to improve the system efficiency is to set $\propto$ as low as possible.

\section{Summery}

Cognitive radio is regarded as the key technology for next generation of wireless network. Dynamic spectrum sharing is one of the most important problems related to Cognitive Radio networks. Based on the competitive spectrum sharing on game theory, an adaptive competitive game and auction-based spectrum sharing mechanism is presented in this chapter. The advantages over the optimal, cooperative and competitive modes have been proved by simulation. A general solution for the instability problem has been proposed and an adaptive method is used for the changing number of secondary users by using cooperative game model when the number of users is small. Another solution to such a problem is presented by using a non-cooperative game model combined with second-price auction to choose a secondary primary user. The decision is based on user reputation and user's valuation of the offered spectrum. We have the solution with maximum total profit and better fairness in spectrum sharing. We have discussed how the increase of competitors would affects the fairness of spectrum sharing and proved that the proposed mechanism offers better revenue to the seller and the bidders in terms of fairness.

\section{References}

[1] FCC, "Spectrum Policy Task Force Report”, ET Docket No. 02-135, pp. 35-53, November 7, 2002.

[2] S.S. Brave, S.B. Deosarkar, and S.A. Bhople, "A Cognitive approach to spectrum sensing in Virtual Unlicensed wireless network", International Conference on Advances in Computing, Communication and Control (ICAC3'09), pp. 668-673, 2009.

[3] M. Buddhikot, “Understanding Dynamic Spectrum Allocation: Models, Taxonomy and Challenges", Proceeding of IEEE DySPAN, 2007.

[4] T. Kamakaris, M. Buddhikot, and R. Iyer, "A Case of Coordinated Dynamic Spectrum Access in Cellular Networks", Proceeding of IEEE DySPAN, 2005.

[5] R. Axelrod, "The Evolution of Cooperation", Basic Books, reprint edition, New York, 1984.

[6] C. Ianculescu, and A. Mudra, "Cognitive Radio and Dynamic Spectrum Sharing", Proceeding of the SDR 05 Technical Conferences and Product Exposition, 2005.

[7] C. Jackson, "Dynamic Sharing of Radio Spectrum: A Brief History", Book chapter, July 2002. Available on line at:

http://ieeexplore.ieee.org/stamp/stamp.jsp?arnumber =01542659 [Accessed March 2010].

[8] Federal Communications Commission, "Spectrum Policy Task Force Report", Report ET Docket, no. 02-135, Nov. 2002. 
[9] 47 CFR 22.501 - Scope. Code of Federal Regulations-Title 47: Telecommunications (December 2005), Available online at:

http:/ / cfr.vlex.com/vid/22-501-scope-19849829 [Accessed March 2010].

[10] A.V. Oppenheim, W.R. Schafer, and R.J. Buck, “Discrete-Time Signal Processing", Prentice-Hall Signal Processing Series, 2nd edition, 1999.

[11] R.V. Cox, C.A. Kamm, L.R. Rabiner, J. Schroeter, and J. G.Wilpon, "Speech and Language Processing for Next-Millennium Communications Services", Proceedings of the IEEE, vol. 88, no. 8, pp. 1314-1337, August 2000.

[12] L. Rabiner, B.H. Juang and M.M. Sondhi, "Digital Speech Processing", Encyclopaedia of Physical Science and Technology, Third Edition, vol. 4, pp. 485-500, 2002.

[13] J.H. McClellan and C.M. Rader, "Number Theory in Digital Signal Processing", Prentice-Hall, Inc., Englewood Cliffs, N.J., 1979.

[14] J.H. McClellan, R.W. Schafer and M.A. Yoder, "Signal Processing First: A Multimedia Approach", Upper Saddle River, NJ: Prentice-Hall, 1998.

[15] B. Schaller, "The Origin, Nature, and Implications of "MOORE'S LAW", the Benchmark of Progress in Semiconductor Electronics", Spring 1996, available online at: http://research.microsoft.com/en-us/um/people/gray/Moore_Law.html [Accessed March 2010].

[16] D. Angel, "Restructuring for Innovation: The Remaking of the U.S. Semiconductor Industry", (New York: The Guilford Press), 1994.

[17] E. Braun, and S. Macdonald, "Revolution in Miniature: The History and Impact of Semiconductor Electronics", (Cambridge: Cambridge University Press), 1982.

[18] A. Dorfman, and S. Nancy," Innovation and Market Structure: Lessons from the Computer and Semiconductor Industries", (Cambridge: Ballinger), 1987.

[19] J. Mitola III, “Cognitive Radio: An Integrated Agent Architecture for Software Defined Radio", PhD Thesis, KTH Royal Institute of Technology, 2000.

[20] R. Axelrod, "The Evolution of Cooperation", Basic Books, reprint edition, New York, 1984.

[21] P.K. Dutta, "Strategies and Games: Theory and Practice”, MIT press, 2001.

[22] S. Haykin, "Cognitive Radio: Brain-empowered Wireless Communications", IEEE Journal in Selected Areas in Communications, vol. 23, pp. 201-220, Feb. 2005.

[23] K. Lee and A. Yener, "Throughput Enhancing Cooperative Spectrum Sensing Strategies for Cognitive Radios", in Proceeding of Asilomar Conference on Signals, Systems and Computers, Pacific Grove, CA, Nov. 2007.

[24] D. Cabric, M. S. Mishra, and R. W. Brodersen, "Implementation Issues in Spectrum Sensing for Cognitive Radios", in Proceeding Asilomar Conference on Signals, Systems, and Computers, Pacific Grove, CA, Nov. 2004.

[25] W. Zhang and K.B. Letaief, "Cooperative Spectrum Sensing With Transmit and Relay Diversity in Cognitive Networks", IEEE Transactions in Wireless Communications, vol. 7, pp. 4761-4766, Dec. 2008.

[26] J. Unnikrishnan and V. Veeravalli, "Cooperative Sensing for Primary Detection in Cognitive Radio", IEEE Journal in Selected Topics in Signal Processing, vol. 2, no. 1, pp. 18-27, Feb. 2008.

[27] B. Wang, K.J.R. Liu, and T. Clancy, "Evolutionary Game Framework for Behavior Dynamics in Cooperative Spectrum Sensing", in Proceeding IEEE Global Communications Conference, New Orleans, LA, Dec. 2008. 
[28] S. Huang, X. Liu, and Z. Ding, “Optimal Sensing-Transmission Structure for Dynamic Spectrum Access", in Proceedings International Conference on Computer Communications (INFOCOM), Rio de Janeiro, Brazil, Apr. 2009.

[29] M. Maskery, V. Krishnamurthy, and Q. Zhao, "Decentralized Dynamic Spectrum Access for Cognitive Radios: Cooperative Design of a Noncooperative Game", IEEE Transaction in Communications, vol. 57, no. 2, pp. 459-469, Feb. 2009.

[30] S. Subranami, T. Basar, S. Armour, D. Kaleshi, and Z. Fan, "Noncooperative Equilibrium Solutions for Spectrum Access in Distributed Cognitive Radio Networks", in Proceeding to IEEE DySPAN, Chicago, IL, Oct. 2008.

[31] M. Bloem, T. Alpcan, and T. Basar, "A Stackelberg Game for Power Control and Channel Allocation in Cognitive Radio Networks", in Proceedings ICST/ACM Game communication Workshop, Nantes, France, Oct. 2007.

[32] S. Sanghavi and B. Hajek, "Optimal Allocation of Divisible Good to Strategic Buyers", The 43rd IEEE Conference on Decision and Control, Paradise Island, Bahamas. December 2004.

[33] O. Raoof, Z. Al-Banna, and H.S. Al-Raweshidy, "Competitive Spectrum Sharing in Wireless Networks: A Dynamic Non-cooperative Game Approach", Springer. Boston, vol. 308/2009, pp. 197-207, August 2009.

[34] O. Raoof, Z. Al-Banna, and H.S. Al-Raweshidy, "A Dynamic Non-cooperative Game Approach for Competitive Spectrum Sharing in Wireless Networks", 15th EUNICE International Workshop - The Internet of the Future. Barcelona, Spain, September 2009.

[35] FCC, "Facilitating Opportunities for Flexible, Efficient, and Reliable Spectrum Use Employing Cognitive Radio Technologies, Notice of Proposed Rulemaking", 03322A1, Dec 2003. Available online at: www.cs.ucdavis.edu/ liu/289I/Material/FCC-03-322A1.pdf [Accessed March 2010].

[36] Q. Zhao, L. Tong, A. Swami, and Y. Chen, "Decentralized Cognitive MAC for Opportunistic Spectrum Access in Ad Hoc Networks: A POMDP Framework", IEEE Journal on Selected Areas in Communication (JSAC), April 2007.

[37] C. Peng, H. Zheng, and B. Zhao, "Utilization and Fairness in Spectrum Assignment for Opportunistic Spectrum Access", ACM Journal of Mobile Networks and Applications (MONET), August 2006.

[38] R. Etkin, A. Parekh, and D. Tse, "Spectrum Sharing for Unlicensed Bands", IEEE Journal on Selected Areas in Communication (JSAC), vol. 25, Iss. 3, pp. 517, April 2007.

[39] T. Alpcan, T. Bas,ar, R. Srikant, and E. Altman, “CDMA Uplink Power Control as a Noncooperative Game", Wireless Networks, vol. 8, pp. 659-670, 2002

[40] J. Huang, R. Berry, and M. Honig, “Distributed Interference Compensation for Wireless Networks", IEEE Journal on Selected Areas in Communication (JSAC), May 2006.

[41] N. Nie, and C. Comaniciu, "Adaptive Channel Allocation Spectrum Etiquette for Congnitive Radio Networks", 1st IEEE Conference on Dynamic Spectrum Access Network (DySPAN), Nov. 2005.

[42] M. Felegyhazi, M. Cagali, S. Bidokhti, and J. Hubaux, "Non-Cooperative Multi-Radio Channel Allocation in Wireless Networks", 26th IEEE conference on Computer Communications (INFOCOM), May 2007. 
[43] I. Hoang, and Y. Liang, "Maximizing Spectrum Utilization of Cognitive Radio Networks Using Channel Allocation and Power Control", IEEE Vehicular Technology Conference (VTC), Sep. 2006.

[44] T. Basar, and G. Olsder, "Dynamic Noncooperative Game Theory", Series in Classics in Applied Mathematics, SIAM, Philadelphia, 2nd Ed, 1999.

[45] B. Platt, J. Price, and H. Tappen, "Pay-to-Bid Auctions", (July 9, 2009), Available at SSRN: http:/ / ssrn.com/abstract=1432169 [Accessed April 2010].

[46] S. H. Chun, and R.J. La, "Auction Mechanism for Spectrum Allocation and Profit Sharing", Game Theory for Networks, GameNets '09., pp. 498-507, 13-15, May 2009.

[47] W.W. Sharkey, F. Beltran, and M. Bykowsky, "Computational analysis of an auction for licensed and unlicensed use of spectrum", Game Theory for Networks, 2009. GameNets '09, pp. 488-497, 13-15, May 2009.

[48] D. Niyato, and E. Hossain, "Market-Equilibrium, Competitive, and Cooperative Pricing for Spectrum Sharing in Cognitive Radio Networks: Analysis and Comparison", IEEE Transactions on Wireless Communications, vol. 7, no. 11, pp. 4273-4283, November 2008.

[49] D. Niyato, and E. Hossain, "Competitive spectrum sharing in cognitive radio networks: a dynamic game approach", IEEE Transactions on Wireless Communications, vol. 7, no. 7, pp. 2651-2660, July 2008.

[50] D. Niyato, E. Hossain, and L. Long, "Competitive Spectrum Sharing and Pricing in Cognitive Wireless Mesh Networks", in Proceedings of IEEE WCNC 2008, Las Vegas, NV, 31 March-3, April 2008.

[51] S.L. Chen, W.A. Cui, Y.F. Luo, and X.H. Yang, "Optimal Design of Online Auctions with Shill Bidding and Open Reserve Price", WiCOM '08. 4th International Conference on Wireless Communications Networking and Mobile Computing, pp. 1-4, 12-14, October 2008.

[52] H.J. Parrsch and J. Robert, "Testing Equilibrium Behaivour At First-Price, Sealed-Bid Auctions With Discrete Bid Increments", Scientific series, Montreal, June 2003.

[53] F.M. Menezes and P.K. Monteiro, "An Introduction to Auction Theory", Oxford Scholarship Online, November 2004. 


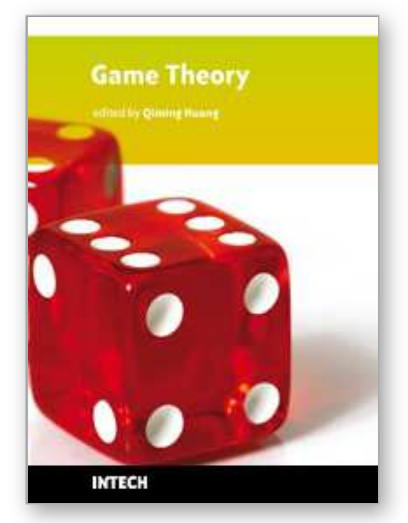

\author{
Game Theory \\ Edited by Qiming Huang
}

ISBN 978-953-307-132-9

Hard cover, 176 pages

Publisher Sciyo

Published online 27, September, 2010

Published in print edition September, 2010

Game theory provides a powerful mathematical framework that can accommodate the preferences and requirements of various stakeholders in a given process as regards the outcome of the process. The chapters' contents in this book will give an impetus to the application of game theory to the modeling and analysis of modern communication, biology engineering, transportation, etc...

\title{
How to reference
}

In order to correctly reference this scholarly work, feel free to copy and paste the following:

Omar Raoof and Hamed Al-Raweshidy (2010). Auction and Game-based Spectrum Sharing in Cognitive Radio Networks, Game Theory, Qiming Huang (Ed.), ISBN: 978-953-307-132-9, InTech, Available from: http://www.intechopen.com/books/game-theory/game-application-in-cognitive-radio-networks

\section{INTECH}

open science | open minds

\section{InTech Europe}

University Campus STeP Ri

Slavka Krautzeka 83/A

51000 Rijeka, Croatia

Phone: +385 (51) 770447

Fax: +385 (51) 686166

www.intechopen.com

\section{InTech China}

Unit 405, Office Block, Hotel Equatorial Shanghai

No.65, Yan An Road (West), Shanghai, 200040, China

中国上海市延安西路65号上海国际贵都大饭店办公楼405单元

Phone: +86-21-62489820

Fax: $+86-21-62489821$ 
(C) 2010 The Author(s). Licensee IntechOpen. This chapter is distributed under the terms of the Creative Commons Attribution-NonCommercialShareAlike-3.0 License, which permits use, distribution and reproduction for non-commercial purposes, provided the original is properly cited and derivative works building on this content are distributed under the same license. 